\title{
Systematic review of the appropriateness of eye care delivery in eye care practice
}

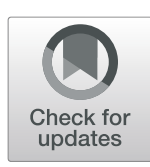

Kam Chun Ho ${ }^{1,2}$, Fiona Stapleton', Louise Wiles ${ }^{1,3,4}$, Peter Hibbert ${ }^{1,3,4}$, Sally Alkhawajah ${ }^{1,5}$, Andrew White ${ }^{1,6,7}$ and Isabelle Jalbert ${ }^{1 *}$ (D)

\begin{abstract}
Background: Health care systems are continually being reformed, however care improvement and intervention effectiveness are often assumed, not measured. This paper aimed to review findings from published studies about the appropriateness of eye care delivery, using existing published evidence and/or experts' practice and to describe the methods used to measure appropriateness of eye care.

Methods: A systematic search was conducted using Medline, Embase and CINAHL (2006 to September 2016). Studies reporting the processes of eye care delivery against existing published evidence and/or experts' practice were selected. Data was extracted from published reports and the methodological quality using a modified critical appraisal tool. The primary outcomes were percentage of appropriateness of eye care delivery. This study was registered with PROSPERO, reference CRD42016049974.

Results: Fifty-seven studies were included. Most studies assessed glaucoma and diabetic retinopathy and the overall methodological quality for most studies was moderate. The ranges of appropriateness of care delivery were $2-100 \%$ for glaucoma, $0-100 \%$ for diabetic retinopathy and 0-100\% for other miscellaneous conditions. Published studies assessed a single ocular condition, a sample from a single centre or a single domain of care, but no study has attempted to measure the overall appropriateness of eye care delivery.

Conclusions: These findings indicated a wide range of appropriateness of eye care delivery, for glaucoma and diabetic eye care. Future research would benefit from a comprehensive approach where appropriateness of eye care is measured across multiple conditions with a single methodology, to guide priorities within eye care delivery and monitor quality improvement initiatives.
\end{abstract}

Keywords: Glaucoma, Delivery of health care, Diabetic retinopathy, Public health, Evidence-based practice, Process assessment (health care)

\section{Background}

Globally, 285 million people of all ages suffer from visual impairment [1]. Long-term ocular conditions, including both ocular diseases (e.g. glaucoma, diabetic retinopathy, age-related macular degeneration and cataract) and uncorrected refractive errors are the major causes of visual impairment worldwide [2]. The prevalence of vision problems is strongly associated with ageing and this compromised visual function affects individuals' ability to perform activities of daily living [3]. Common eye

\footnotetext{
* Correspondence: i.jalbert@unsw.edu.au

'School of Optometry and Vision Science, UNSW Sydney, Sydney, NSW 2052, Australia

Full list of author information is available at the end of the article
}

diseases can often be detected early and their visual impact minimised or they can be prevented by appropriate eye care services, including routine eye examinations [46]. Due to the growing demand for eye care in the context of resource scarcity, interest in measuring and improving the appropriateness of eye care delivery is growing $[7,8]$. Appropriate care is defined as provision of evidence-based care that is relevant to the patient's needs and based on established standards [9].

Translation of best available evidence into clinical practice is important, ensuring that both efficacy and cost-effectiveness of patient management is maintained [10]. Evidence-based guidelines aim to translate well conducted scientific trials into easy to apply 
recommendations. Such guidelines intend to guide practitioners and help them to improve their professional practice and optimize patient care [11]. Evidence-based guidelines are not always adhered to and/or fully implemented in the clinical setting. Adherence to guidelines can be quantitatively measured using quality indicators of appropriateness of care delivery. Quality Indicators can be defined as "measurable components of a standard or guideline, with explicit criteria for inclusion, exclusion, time frame, setting and compliance action" [12].

Evidence of suboptimal care being delivered exist, arising from several large studies assessing appropriateness of care across different health conditions. The RAND study conducted in 2000 in the United States evaluated performance on 439 quality indicators of appropriateness of care for 30 acute and chronic conditions as well as preventive care. The RAND study showed that American adults received recommended care only 55\% (range 11-79\%) of the time [13]. More recently, the CareTrack study in Australia showed similar results with 57\% (range 13-90\%) of Australian adults receiving appropriate care across 22 conditions [12]. Ocular conditions were not included in the CareTrack study [12]. Defining existing eye care practice patterns and current variation from best practices is an important component of a systemic approach to improving appropriateness of eye care $[14,15]$.

\section{Purpose}

This paper aimed to review findings from published studies about the appropriateness of eye care delivery, using existing published evidence and/or experts' practice. A secondary aim was to describe and compare the variety of methods used to measure appropriateness of eye care.

\section{Methods}

\section{Data sources and searches}

A systematic search was conducted using Medline, Embase and the Cumulative Index to Nursing and Allied Health Literature (CINAHL) electronic databases to identify studies related to the appropriateness of eye care. The search strategy was reviewed and tested by an academic librarian and reviewed by content experts (IJ and FS). The literature review process followed the Preferred Reporting Items for Systematic reviews and MetaAnalyses (PRISMA) procedures [16] and the review protocol was published on PROSPERO (http://www.crd. york.ac.uk/prospero/, reference CRD42016049974). As eye conditions with higher prevalence and heavier burden on the health system, the emphasis was put on glaucoma, diabetic retinopathy, refractive error, cataract and macular degeneration [17]. The search incorporated the three elements:
1) Profession-specific terms: "Optometr"”, "Ophthalmolog"", "General practitioner", "Orthopt"," "Ophthalmic nurse" "," "Ophthalmic practitioner".

2) Subject headings: Exp"Quality of Health Care" in Medline, Exp"Health care quality" in Embase, MH"Health Services Research+" in CINAHL.

3) Condition-specific terms: Exp Glaucoma, Exp diabetic retinopathy, Exp refractive errors, Exp macular degeneration, Exp cataract.

An example of the full electronic search strategy for Medline database is illustrated in Additional file 1.

\section{Study selection}

Reference lists and citations were used to cross-check the results of our search. The reference details and abstracts of the 5596 articles retrieved from the literature search after duplicates removal were reviewed by one reviewer $(\mathrm{KCH})$. Studies assessing the processes of eye care delivery against existing published evidence and experts' practice (e.g. consultant ophthalmologists' practice) were included. Studies assessing outcomes of care delivery such as patient satisfaction or those assessing structural aspects of care delivery such as workforce characteristics, infrastructure, regulations and policies were excluded from analysis in this review. The search was not restricted by type of study design, and no other limitations (e.g. population, intervention, comparison, length of follow-up) were set. The search was limited to English and 10 years to the search date (2006 to 16th September 2016). Studies conducted more than 10 years ago were excluded, on the basis that appropriateness of care was likely to change over time, and that older studies might not reflect recent changes in care delivery standards [18]. The references were narrowed to 65 articles after title and abstract screening following the application of exclusion criteria (Fig. 1). A further six articles were excluded after full text review with three that did not access process of care and three that did not measure against existing published evidence or experts' practice.

\section{Data extraction and quality assessment}

Each paper was reviewed and information was extracted based on the following characteristics:

- Country

- Condition(s) - the eye condition(s) for which the appropriateness of care was assessed

- Professions - the health professions delivering the care of the assessed eye condition

- Methods - the method used to assess the appropriateness of eye care delivered 


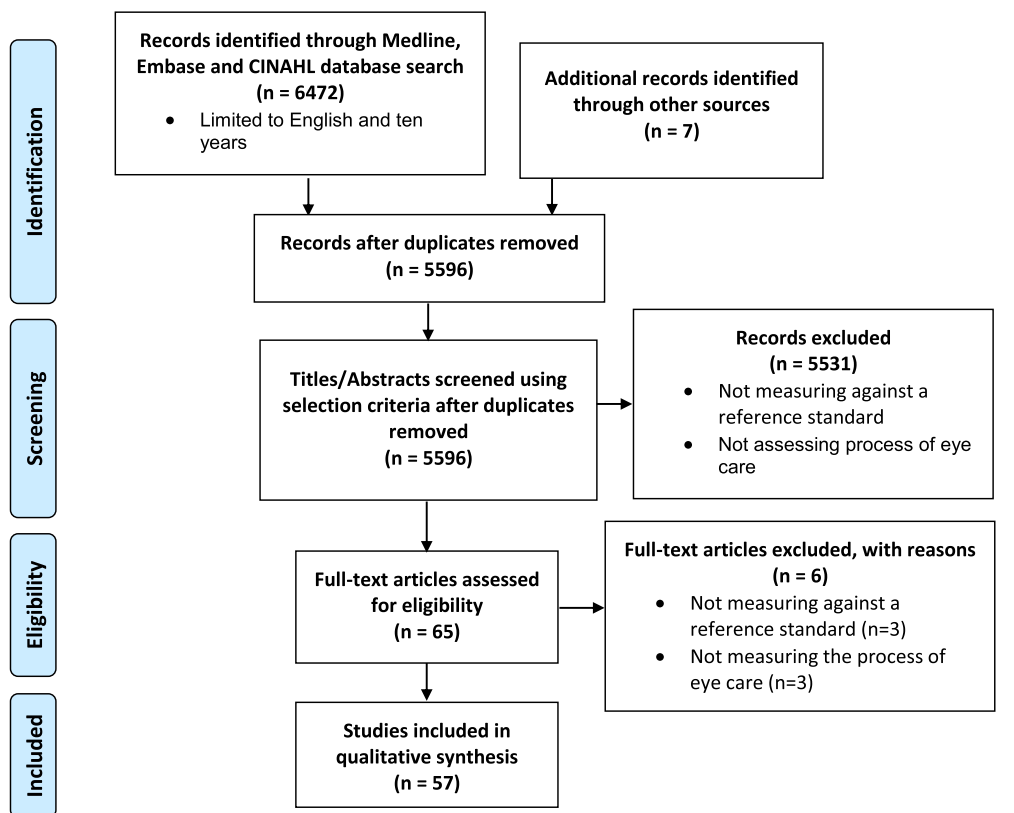

Fig. 1 PRISMA flow diagram for appropriateness of eye care delivery

- Sample size

- Response rate

- Evidence sources - the reference standard used to assess the appropriateness of eye care delivered

- Settings - classification based on whether study was conducted in hospital or independent practice

- Number of sites - the number of sites that the study was conducted at

- Timing - the timing and visit types assessed in the article (e.g. at diagnosis, follow-up, etc)

- Percentage of encounters with appropriate eye care - the number of quality indicators met over the total number of relevant quality indicators

Taking into consideration the diversity of study types (e.g. descriptive, interventional and observational studies, record reviews, and surveys), two reviewers $(\mathrm{KCH}$ and SA) independently assessed the quality of each article using a validated critical appraisal tool [19]. The applied tool was modified by adding questions from other validated critical appraisal tools including Critical Appraisal Skills Programme (CASP) diagnostic checklist [20], National Institutes of Health (NIH) Quality Assessment Tool For Observational Cohort And Cross-Sectional Studies [21], Joanna Briggs Institute (JBI) Critical Appraisal Checklist For Studies Reporting Prevalence Data [22], Effective Public Health Practice Project (EPHPP) Quality assessment tool for quantitative studies [23].

The modified quality assessment tool included 17 individual criterions with questions from validated critical appraisal tools [20-23] (Additional file 2) and grouped in the seven categories listed below:

- Quality of reporting (adequate description of the context [19], clearly stated aims [19-21], eligibility [21], methods and findings [20])

- Selection bias (representative of the selected individuals [22, 23], response rate at least 50\% [21], and sample size justification [21])

- Study design (presence of randomisation [23], presence of control group [19, 23])

- Blinding (blinding of outcome assessors to the intervention or exposure status of participants [20, 21, 23], blinding of participants to research question [23], and blinding of decision making between participants and experts [20])

- Data collection tools (reliability of the data collection tool $[22,23]$ and valid reference used to assess the appropriateness of care [20])

- Analysis (sufficient rigorous data analysis [19, 22, 23])

- Limitations (key potential confounders are identified and accounted for [21-23])

The number of criteria used varied depending on the study design of the publication being reviewed. An overall rating was allocated for each paper as a percentage based on the number of criteria met over the number of relevant criteria for the corresponding study design. If less than $60 \%$ criteria relevant to the study design was 
met, this item was scored as Weak in the quality assessment tool. It was scored moderate if $60-79 \%$ of criteria were met and strong if $80-100 \%$ of criteria were met. A third reviewer (IJ) resolved any disagreements and consensus was reached through discussion. All articles were included, and the results of critical appraisal are provided in Additional file 3.

\section{Data synthesis and analysis}

Due to the anticipated heterogeneity of included studies, no plans were made to pool the results statistically, therefore a meta-analysis was not undertaken. For each study, the range of percentage of appropriate care (summary data from published reports, but not individual patient-level data) and the number of quality indicators were separated according to the nature of the quality indicators into the following six domains of care: 'history taking', 'physical examination', 'management', 'recall period', 'referral' and 'patient education'. On occasion, data provided in the papers had to be reclassified to fit these proposed domains of care. Data were also reanalysed as required so that the results could be presented in terms of appropriateness to prescribed care and not the reverse (i.e. percentage with inappropriate care).

\section{Results}

Of 6472 citations, 57 articles met the inclusion (see Fig. 1 ). The characteristics of these studies are presented in Table 1. The majority of the studies were from the United Kingdom (UK) $(n=25)$ and the United States of America (USA) $(n=15)$, with Australia $(n=5)$, Australia and New Zealand (NZ) $(n=2)$ and other countries accounting for the remainder. Among the 57 papers, twothirds examined eye care delivery for glaucoma $(n=28)$ and diabetic retinopathy $(n=11)$. The majority of papers assessed the care delivered by optometrists $(n=22)$ and ophthalmologists $(n=19)$, with another seven studies including both professions. Half of the studies were rated moderate $(60-79 \%$ of quality criteria met) for the methodological quality $(n=29)$, another one-third were rated strong $(80-100 \%$ of quality criteria met) $(n=19)$ and the remainder were rated weak $(<60 \%$ of quality criteria met) $(n=9)$. For all conditions but diabetic retinopathy, a similar pattern of distribution of methodological quality (i.e. mostly moderate) was observed. However, for diabetic retinopathy most of the studies (73\%) were rated strong in methodological quality.

Record review (26 of 57 studies) and practitioner survey with or without case vignettes (15 of 57 studies) were the most commonly used methods, with one study using a combination of both methods and one study using both methods with claims data and patient survey. When eye care appropriateness was measured using record review, assessments were most frequently conducted at a single site $(n=19)$ and in these cases, studies were conducted in a hospital setting (Fig. 2). Use of a single site reduces logistical challenges, but the results may not be generalisable to other environments with a different location, business models and case-mix. For example, the record review conducted in the Department of Veterans Affairs, which caters to a population that is predominantly male, may not be generalised to clinic settings and patient populations outside the Veterans Affairs system [50].

Appropriateness of eye care was generally measured as compliance against scientific evidence or consensus with clinical experts in the field with around two-thirds of the articles having measured eye care appropriateness against recommendations from clinical practice guidelines $(n=38)$ and $16 \%$ having used experts' opinions $(n=9)$.

A small number of studies measured eye care appropriateness against expert care rather than against clinical practice guidelines, where the same patients are examined twice, once by the practitioners and once by experts $[36,135,143]$.

Eye care appropriateness results are summarized in Table 2. It is important to note at the outset that the timing (e.g. once during a period, at the diagnosis visit, etc.), type of visits (e.g. first visit, follow-up visit, etc.), the health professions and settings assessed, and the method used to collect the data (e.g. record review) vary between studies (see Table 2) and may confound the appropriateness of eye care results.

Twenty-eight studies reporting on eye care appropriateness in glaucoma screening, glaucoma suspects and/ or glaucoma patients were included. In more than half of the studies (15 of 28), the appropriateness of glaucoma care was measured via a review of hospital records. Appropriate 'management' and 'recall period' for glaucoma were reported most of the time, whereas 'physical examination' and 'referral' for glaucoma were not delivered as appropriately at times (Fig. 3a and b). Overall, the appropriateness of glaucoma care ranged widely from 2 to $100 \%$. The appropriateness of glaucoma care assessed using clinical agreement with experts was the only method where appropriate care was delivered consistently at least $50 \%$ of the time. Although studies investigated the appropriateness of glaucoma delivered by optometrists and ophthalmologists, no obvious differences between professions were noted.

Eleven studies have reported on appropriateness of eye care delivery in diabetic patients. Overall, diabetes eye care compliance also ranged widely from 0 to $100 \%$. That wide range and the relatively small number of studies available makes it challenging to detect obvious patterns in individual domains for diabetes care (Fig. 3c and d). For example, only a single study 
Table 1 Study Characteristics $(n=57)$. USA = United States of America, UK=United Kingdom, NZ= New Zealand, A\&E = accident and emergency

\begin{tabular}{|c|c|c|c|c|c|c|c|c|}
\hline Country & Evidence sources & Year & Professions & Outcomes & Methods & $\begin{array}{l}\text { Overall } \\
\text { quality }^{a}\end{array}$ & $\begin{array}{l}\text { Author } \\
\text { (reference) }\end{array}$ & $n^{b}$ \\
\hline \multicolumn{9}{|l|}{ Glaucoma } \\
\hline UK & $\begin{array}{l}\text { Clinical practice } \\
\text { guidelines }[24,25]\end{array}$ & 2013 & Ophthalmologist & $\begin{array}{l}\text { Current } \\
\text { practice } \\
\text { pattern }\end{array}$ & Record review & Strong & Fung et al. [26] & 101 \\
\hline UK & $\begin{array}{l}\text { Clinical practice } \\
\text { guidelines [25] }\end{array}$ & 2012 & $\begin{array}{l}\text { Ophthalmologist } \\
\text { \& optometrist }\end{array}$ & $\begin{array}{l}\text { Guidelines } \\
\text { adherence }\end{array}$ & Record review & Weak & $\begin{array}{l}\text { Chawla et al. } \\
\text { [27] }\end{array}$ & 200 \\
\hline UK & $\begin{array}{l}\text { Clinical practice } \\
\text { guidelines }[25,28]\end{array}$ & 2012 & Optometrist & $\begin{array}{l}\text { Guidelines } \\
\text { adherence }\end{array}$ & Record review & Moderate & Khan et al. [29] & 114 \\
\hline UK & $\begin{array}{l}\text { Clinical practice } \\
\text { guidelines [30] }\end{array}$ & 2012 & Optometrist & $\begin{array}{l}\text { Validation of } \\
\text { self-reported } \\
\text { practice }\end{array}$ & $\begin{array}{l}\text { Interview with practitioner } \\
\text { and unannounced } \\
\text { standardised patient }\end{array}$ & Moderate & $\begin{array}{l}\text { Theodossiades } \\
\text { et al. [31] }\end{array}$ & 34 \\
\hline UK & $\begin{array}{l}\text { Clinical practice } \\
\text { guidelines [25] }\end{array}$ & 2011 & Ophthalmologist & $\begin{array}{l}\text { Current } \\
\text { practice } \\
\text { pattern }\end{array}$ & Practitioner Survey & Moderate & $\begin{array}{l}\text { Stead et al. } \\
\text { [32] }\end{array}$ & $\begin{array}{l}626 \\
(69 \%)\end{array}$ \\
\hline UK & $\begin{array}{l}\text { Clinical practice } \\
\text { guidelines }[33,34]\end{array}$ & 2009 & Optometrist & $\begin{array}{l}\text { Quality of } \\
\text { referral letter }\end{array}$ & Record review & Moderate & $\begin{array}{l}\text { Scully et al. } \\
\text { [35] }\end{array}$ & 121 \\
\hline UK & Experts' opinions & 2012 & Optometrist & $\begin{array}{l}\text { Diagnostic } \\
\text { accuracy }\end{array}$ & $\begin{array}{l}\text { Clinical agreement with } \\
\text { expert }\end{array}$ & Moderate & $\begin{array}{l}\text { Marks et al. } \\
{[36]}\end{array}$ & 145 \\
\hline UK & Experts' opinions & 2011 & Optometrist & $\begin{array}{l}\text { Diagnostic } \\
\text { accuracy }\end{array}$ & Record review & Moderate & $\begin{array}{l}\text { Ho and Vernon } \\
\text { [37] }\end{array}$ & 140 \\
\hline UK & Experts' opinions & 2011 & Optometrist & $\begin{array}{l}\text { Quality of } \\
\text { referral }\end{array}$ & Record review & Moderate & $\begin{array}{l}\text { Shah and } \\
\text { Murdoch [38] }\end{array}$ & 110 \\
\hline UK & Experts' opinions & 2010 & Optometrist & $\begin{array}{l}\text { Feasibility of } \\
\text { shared care }\end{array}$ & Record review & Strong & Syam et al. [39] & 1184 \\
\hline UK & Experts' opinions & 2010 & Optometrist & $\begin{array}{l}\text { Quality of } \\
\text { referral }\end{array}$ & Record review & Weak & $\begin{array}{l}\text { Lockwood et } \\
\text { al. [40] }\end{array}$ & 441 \\
\hline UK & Experts' opinions & 2007 & $\begin{array}{l}\text { Ophthalmologist } \\
\text { \& optometrist }\end{array}$ & $\begin{array}{l}\text { Diagnostic } \\
\text { accuracy }\end{array}$ & $\begin{array}{l}\text { Clinical agreement with } \\
\text { expert }\end{array}$ & Strong & $\begin{array}{l}\text { Azuara-Blanco } \\
\text { et al. [41] }\end{array}$ & 100 \\
\hline UK & Experts' opinions & 2006 & Optometrist & $\begin{array}{l}\text { Quality of } \\
\text { referral }\end{array}$ & Record review & Weak & Patel et al. [42] & 376 \\
\hline UK & Experts' opinions & 2006 & $\begin{array}{l}\text { Optometrist \& } \\
\text { associate } \\
\text { specialists }\end{array}$ & $\begin{array}{l}\text { Diagnostic } \\
\text { accuracy }\end{array}$ & $\begin{array}{l}\text { Clinical agreement with } \\
\text { expert }\end{array}$ & Moderate & $\begin{array}{l}\text { Banes et al. } \\
\text { [43] }\end{array}$ & 350 \\
\hline USA & $\begin{array}{l}\text { Clinical practice } \\
\text { guidelines }[24,44]\end{array}$ & 2016 & Ophthalmologist & $\begin{array}{l}\text { Current } \\
\text { practice } \\
\text { pattern }\end{array}$ & Record review & Moderate & $\begin{array}{l}\text { Solano- } \\
\text { Moncada et al. } \\
{[45]}\end{array}$ & 250 \\
\hline USA & $\begin{array}{l}\text { Clinical practice } \\
\text { guidelines [44] }\end{array}$ & 2016 & $\begin{array}{l}\text { Ophthalmologist } \\
\text { \& optometrist }\end{array}$ & $\begin{array}{l}\text { Current } \\
\text { practice } \\
\text { pattern }\end{array}$ & Claims data & Strong & Elam et al. [46] & $\begin{array}{l}56 \\
675\end{array}$ \\
\hline USA & $\begin{array}{l}\text { Clinical practice } \\
\text { guidelines [47] }\end{array}$ & 2015 & Ophthalmologist & $\begin{array}{l}\text { Diagnostic } \\
\text { accuracy }\end{array}$ & Record review & Moderate & $\begin{array}{l}\text { Zebardast et } \\
\text { al. [48] }\end{array}$ & 212 \\
\hline USA & $\begin{array}{l}\text { Clinical practice } \\
\text { guidelines [49] \& experts' } \\
\text { opinions }\end{array}$ & 2013 & Ophthalmologist & $\begin{array}{l}\text { Guidelines } \\
\text { adherence }\end{array}$ & Record review & Strong & Ong et al. [50] & 103 \\
\hline USA & $\begin{array}{l}\text { Clinical practice } \\
\text { guidelines [44] }\end{array}$ & 2012 & $\begin{array}{l}\text { Ophthalmologist } \\
\text { \& optometrist }\end{array}$ & $\begin{array}{l}\text { Current } \\
\text { practice } \\
\text { pattern }\end{array}$ & Claims data & Moderate & $\begin{array}{l}\text { Swamy et al. } \\
{[51]}\end{array}$ & $\begin{array}{l}143 \\
374\end{array}$ \\
\hline USA & $\begin{array}{l}\text { Clinical practice } \\
\text { guidelines [49] }\end{array}$ & 2007 & Ophthalmologist & $\begin{array}{l}\text { Guidelines } \\
\text { adherence }\end{array}$ & $\begin{array}{l}\text { Claims data, record review, } \\
\text { practitioner survey and } \\
\text { patient survey }\end{array}$ & Moderate & $\begin{array}{l}\text { Quigley et al. } \\
\text { [52] }\end{array}$ & 300 \\
\hline USA & $\begin{array}{l}\text { Clinical practice } \\
\text { guidelines [53] }\end{array}$ & 2006 & Ophthalmologist & $\begin{array}{l}\text { Current } \\
\text { practice } \\
\text { pattern }\end{array}$ & Claims data & Strong & $\begin{array}{l}\text { Coleman et al. } \\
{[54]}\end{array}$ & 4427 \\
\hline Australia \& & Clinical practice & 2015 & Optometrist & Current & Practitioner Survey with & Moderate & Zangerl et al. & 818 \\
\hline
\end{tabular}


Table 1 Study Characteristics $(n=57)$. USA = United States of America, UK=United Kingdom, NZ = New Zealand, A\&E = accident and emergency (Continued)

\begin{tabular}{|c|c|c|c|c|c|c|c|c|}
\hline Country & Evidence sources & Year & Professions & Outcomes & Methods & $\begin{array}{l}\text { Overall } \\
\text { quality }^{a}\end{array}$ & $\begin{array}{l}\text { Author } \\
\text { (reference) }\end{array}$ & $n^{b}$ \\
\hline NZ & guidelines [55] & & & $\begin{array}{l}\text { practice } \\
\text { pattern }\end{array}$ & case vignette & & {$[56]$} & $(18 \%)$ \\
\hline $\begin{array}{l}\text { Australia \& } \\
\text { NZ }\end{array}$ & $\begin{array}{l}\text { Clinical practice } \\
\text { guidelines }[47,57,58]\end{array}$ & 2008 & Ophthalmologist & $\begin{array}{l}\text { Current } \\
\text { practice } \\
\text { pattern }\end{array}$ & Practitioner Survey & Strong & Liu [59] & $\begin{array}{l}627 \\
(78 \%)\end{array}$ \\
\hline Scotland & $\begin{array}{l}\text { Clinical practice } \\
\text { guidelines }[25,60]\end{array}$ & 2015 & Optometrist & $\begin{array}{l}\text { Quality of } \\
\text { referral }\end{array}$ & Record review & Strong & $\begin{array}{l}\text { El-Assal et al. } \\
\text { [61] }\end{array}$ & 1622 \\
\hline Scotland & $\begin{array}{l}\text { Clinical practice } \\
\text { guidelines [60] }\end{array}$ & 2009 & Optometrist & $\begin{array}{l}\text { Quality of } \\
\text { referral }\end{array}$ & Record review & Moderate & Ang et al. [62] & 303 \\
\hline Canada & $\begin{array}{l}\text { Clinical practice } \\
\text { guidelines [63] }\end{array}$ & 2014 & $\begin{array}{l}\text { Ophthalmologist } \\
\text { \& optometrist }\end{array}$ & $\begin{array}{l}\text { Quality of } \\
\text { referral letter }\end{array}$ & Record review & Moderate & $\begin{array}{l}\text { Cheng et al. } \\
\text { [64] }\end{array}$ & 200 \\
\hline Germany & $\begin{array}{l}\text { Clinical practice } \\
\text { guidelines [57] }\end{array}$ & 2008 & Ophthalmologist & $\begin{array}{l}\text { Guidelines } \\
\text { adherence }\end{array}$ & Practitioner Survey & Moderate & $\begin{array}{l}\text { Vorwerk et al. } \\
\text { [65] }\end{array}$ & $\begin{array}{l}335 \\
(12 \%)\end{array}$ \\
\hline Singapore & $\begin{array}{l}\text { Clinical practice } \\
\text { guidelines [66] }\end{array}$ & 2008 & Ophthalmologist & $\begin{array}{l}\text { Current } \\
\text { practice } \\
\text { pattern }\end{array}$ & Practitioner Survey & Strong & Ang et al. [67] & $\begin{array}{l}126 \\
(80 \%)\end{array}$ \\
\hline \multicolumn{9}{|c|}{ Diabetic retinopathy } \\
\hline Australia & $\begin{array}{l}\text { Clinical practice } \\
\text { guidelines [68] }\end{array}$ & 2011 & Optometrist & $\begin{array}{l}\text { Current } \\
\text { practice } \\
\text { pattern }\end{array}$ & Practitioner Survey & Weak & $\begin{array}{l}\text { Slater and } \\
\text { Chakman [69] }\end{array}$ & $\begin{array}{l}985 \\
(26 \%)\end{array}$ \\
\hline Australia & $\begin{array}{l}\text { Clinical practice } \\
\text { guidelines [70] }\end{array}$ & 2011 & Optometrist & $\begin{array}{l}\text { Current } \\
\text { practice } \\
\text { pattern }\end{array}$ & $\begin{array}{l}\text { Practitioner Survey with } \\
\text { case vignette }\end{array}$ & Strong & Ting et al. [71] & $\begin{array}{l}568 \\
(57 \%)\end{array}$ \\
\hline Australia & $\begin{array}{l}\text { Clinical practice } \\
\text { guidelines [70] }\end{array}$ & 2010 & Ophthalmologist & $\begin{array}{l}\text { Guidelines } \\
\text { adherence }\end{array}$ & $\begin{array}{l}\text { Practitioner Survey with } \\
\text { case vignette }\end{array}$ & Strong & Yuen et al. [72] & $\begin{array}{l}480 \\
(63 \%)\end{array}$ \\
\hline NZ & $\begin{array}{l}\text { Clinical practice } \\
\text { guidelines [73] }\end{array}$ & 2012 & Optometrist & $\begin{array}{l}\text { Guidelines } \\
\text { adherence }\end{array}$ & Record review & Strong & $\begin{array}{l}\text { Hutchins et al. } \\
\text { [74] }\end{array}$ & 157 \\
\hline USA & $\begin{array}{l}\text { Clinical practice } \\
\text { guidelines [75] }\end{array}$ & 2012 & $\begin{array}{l}\text { Ophthalmologist } \\
\text { \& optometrist }\end{array}$ & $\begin{array}{l}\text { Current } \\
\text { practice } \\
\text { pattern }\end{array}$ & Patient survey & Strong & Chou et al. [76] & $\begin{array}{l}29 \\
495\end{array}$ \\
\hline USA & $\begin{array}{l}\text { Clinical practice } \\
\text { guidelines [77] }\end{array}$ & 2010 & Ophthalmologist & $\begin{array}{l}\text { Guidelines } \\
\text { adherence }\end{array}$ & Record review & Strong & $\begin{array}{l}\text { Tseng et al. } \\
{[78]}\end{array}$ & 70 \\
\hline $\begin{array}{l}\text { Hong } \\
\text { Kong }\end{array}$ & $\begin{array}{l}\text { Clinical practice } \\
\text { guidelines [79] }\end{array}$ & 2016 & $\begin{array}{l}\text { General } \\
\text { practitioner }\end{array}$ & $\begin{array}{l}\text { Guidelines } \\
\text { adherence }\end{array}$ & Practitioner Survey & Strong & $\begin{array}{l}\text { Wong et al. } \\
{[80]}\end{array}$ & $\begin{array}{l}414 \\
(13 \%)\end{array}$ \\
\hline Bahrain & $\begin{array}{l}\text { Clinical practice } \\
\text { guidelines [81] }\end{array}$ & 2014 & $\begin{array}{l}\text { General } \\
\text { practitioner }\end{array}$ & $\begin{array}{l}\text { Guidelines } \\
\text { adherence }\end{array}$ & Record review & Strong & $\begin{array}{l}\text { Al-Ubaidi et al. } \\
\text { [82] }\end{array}$ & 200 \\
\hline Switzerland & $\begin{array}{l}\text { Clinical practice } \\
\text { guidelines [83] }\end{array}$ & 2013 & $\begin{array}{l}\text { General } \\
\text { practitioner }\end{array}$ & $\begin{array}{l}\text { Guidelines } \\
\text { adherence }\end{array}$ & Record review & Moderate & $\begin{array}{l}\text { Burgmann et } \\
\text { al. [84] }\end{array}$ & 275 \\
\hline UK & $\begin{array}{l}\text { Clinical practice } \\
\text { guidelines [85] }\end{array}$ & 2011 & $\begin{array}{l}\text { General } \\
\text { practitioner }\end{array}$ & $\begin{array}{l}\text { Guidelines } \\
\text { adherence }\end{array}$ & Record review & Strong & $\begin{array}{l}\text { Mc Hugh et al. } \\
\text { [86] }\end{array}$ & 3010 \\
\hline Brazil & $\begin{array}{l}\text { Clinical practice } \\
\text { guidelines [87] }\end{array}$ & 2007 & $\begin{array}{l}\text { General } \\
\text { practitioner }\end{array}$ & $\begin{array}{l}\text { Current } \\
\text { practice } \\
\text { pattern }\end{array}$ & Practitioner Survey & Weak & Preti et al. [88] & $\begin{array}{l}168 \\
(34 \%)\end{array}$ \\
\hline \multicolumn{9}{|c|}{ Age-related macular degeneration } \\
\hline Italy & $\begin{array}{l}\text { Multiple clinical trials [89- } \\
\text { 92] }\end{array}$ & 2016 & Ophthalmologist & $\begin{array}{l}\text { Guidelines } \\
\text { adherence }\end{array}$ & Interview with patient & Moderate & $\begin{array}{l}\text { Parodi et al. } \\
\text { [93] }\end{array}$ & 283 \\
\hline Turkey & $\begin{array}{l}\text { Multiple clinical trials [89, } \\
90,94]\end{array}$ & 2015 & Ophthalmologist & $\begin{array}{l}\text { Current } \\
\text { practice } \\
\text { pattern }\end{array}$ & Practitioner Survey & Moderate & $\begin{array}{l}\text { Muhammed et } \\
\text { al. [95] }\end{array}$ & $\begin{array}{l}249 \\
(21 \%)\end{array}$ \\
\hline UK & $\begin{array}{l}\text { Multiple clinical trials [89, } \\
\text { 96-99] }\end{array}$ & 2013 & $\begin{array}{l}\text { Ophthalmologist } \\
\text { \& optometrist }\end{array}$ & $\begin{array}{l}\text { Current } \\
\text { practice } \\
\text { pattern }\end{array}$ & $\begin{array}{l}\text { Practitioner Survey with } \\
\text { case vignette }\end{array}$ & Weak & $\begin{array}{l}\text { Lawrenson } \\
\text { and Evans } \\
{[100]}\end{array}$ & $\begin{array}{l}1468 \\
(15 \%)\end{array}$ \\
\hline USA & Multiple clinical trials $[89$, & 2008 & Ophthalmologist & Current & Patient survey & Moderate & Charkoudian et & 332 \\
\hline
\end{tabular}


Table 1 Study Characteristics ( $n=57)$. USA = United States of America, UK=United Kingdom, NZ = New Zealand, A\&E =accident and emergency (Continued)

\begin{tabular}{|c|c|c|c|c|c|c|c|c|}
\hline Country & Evidence sources & Year & Professions & Outcomes & Methods & $\begin{array}{l}\text { Overall } \\
\text { quality }^{\mathrm{a}}\end{array}$ & $\begin{array}{l}\text { Author } \\
\text { (reference) }\end{array}$ & $n^{b}$ \\
\hline & $101,102]$ & & & $\begin{array}{l}\text { practice } \\
\text { pattern }\end{array}$ & & & al. [103] & $(99 \%)$ \\
\hline \multicolumn{9}{|l|}{ Cataract } \\
\hline UK & $\begin{array}{l}\text { Clinical practice } \\
\text { guidelines [104] }\end{array}$ & 2011 & Ophthalmologist & $\begin{array}{l}\text { Current } \\
\text { practice } \\
\text { pattern }\end{array}$ & Practitioner Survey & Weak & $\begin{array}{l}\text { Gomaa and } \\
\text { Liu [105] }\end{array}$ & $\begin{array}{l}158 \\
(53 \%)\end{array}$ \\
\hline UK & $\begin{array}{l}\text { Clinical practice } \\
\text { guidelines [106] }\end{array}$ & 2009 & $\begin{array}{l}\text { Optometrist \& } \\
\text { general } \\
\text { practitioner }\end{array}$ & $\begin{array}{l}\text { Quality of } \\
\text { referral letter }\end{array}$ & Record review & Strong & Park et al. [107] & 124 \\
\hline UK & $\begin{array}{l}\text { Clinical practice } \\
\text { guidelines [108] }\end{array}$ & 2006 & Optometrist & $\begin{array}{l}\text { Quality of } \\
\text { referral letter }\end{array}$ & Record review & Moderate & $\begin{array}{l}\text { Lash et al. } \\
\text { [109] }\end{array}$ & 351 \\
\hline USA & $\begin{array}{l}\text { Clinical practice } \\
\text { guidelines [110] }\end{array}$ & 2009 & $\begin{array}{l}\text { Resident } \\
\text { ophthalmologist }\end{array}$ & $\begin{array}{l}\text { Guidelines } \\
\text { adherence }\end{array}$ & Record review & Strong & $\begin{array}{l}\text { Niemiec et al. } \\
{[1111]}\end{array}$ & 129 \\
\hline \multicolumn{9}{|c|}{ Preventative eye care } \\
\hline UK & $\begin{array}{l}\text { Clinical practice } \\
\text { guidelines [112-114] \& } \\
\text { experts' opinions }\end{array}$ & 2009 & Optometrist & $\begin{array}{l}\text { Current } \\
\text { practice } \\
\text { pattern }\end{array}$ & $\begin{array}{l}\text { Unannounced Standardised } \\
\text { patient }\end{array}$ & Moderate & $\begin{array}{l}\text { Shah et al. } \\
\text { [115] }\end{array}$ & 100 \\
\hline UK & $\begin{array}{l}\text { Clinical practice } \\
\text { guidelines }[114,116,117] \\
\text { \& experts' opinions }\end{array}$ & 2009 & Optometrist & $\begin{array}{l}\text { Current } \\
\text { practice } \\
\text { pattern }\end{array}$ & $\begin{array}{l}\text { Unannounced Standardised } \\
\text { patient }\end{array}$ & Moderate & $\begin{array}{l}\text { Shah et al. } \\
\text { [118] }\end{array}$ & 102 \\
\hline UK & $\begin{array}{l}\text { Clinical practice } \\
\text { guidelines }[114,117,119] \\
\text { \& experts' opinions }\end{array}$ & 2008 & Optometrist & $\begin{array}{l}\text { Current } \\
\text { practice } \\
\text { pattern }\end{array}$ & $\begin{array}{l}\text { Unannounced Standardised } \\
\text { patient }\end{array}$ & Moderate & $\begin{array}{l}\text { Shah et al. } \\
\text { [120] }\end{array}$ & 100 \\
\hline Australia & $\begin{array}{l}\text { Multiple clinical trials' } \\
\text { results }[89,90,121-128]\end{array}$ & 2015 & Optometrist & $\begin{array}{l}\text { Current } \\
\text { practice } \\
\text { pattern }\end{array}$ & Practitioner Survey & Moderate & $\begin{array}{l}\text { Downie and } \\
\text { Keller [129] }\end{array}$ & $\begin{array}{l}283 \\
(6.7 \%)\end{array}$ \\
\hline \multicolumn{9}{|l|}{ Dry eye } \\
\hline Australia & $\begin{array}{l}\text { Clinical practice } \\
\text { guidelines }[130,131]\end{array}$ & 2013 & Optometrist & $\begin{array}{l}\text { Guidelines } \\
\text { adherence }\end{array}$ & Practitioner Survey & Moderate & $\begin{array}{l}\text { Downie et al. } \\
\text { [132] }\end{array}$ & $\begin{array}{l}144 \\
(22 \%)\end{array}$ \\
\hline USA & $\begin{array}{l}\text { Clinical practice } \\
\text { guidelines [133] }\end{array}$ & 2010 & Ophthalmologist & $\begin{array}{l}\text { Guidelines } \\
\text { adherence }\end{array}$ & Record review & Weak & Lin et al. [134] & 178 \\
\hline \multicolumn{9}{|c|}{ All ocular conditions at A\&E } \\
\hline UK & Experts' opinions & 2007 & Optometrist & $\begin{array}{l}\text { Diagnostic } \\
\text { accuracy }\end{array}$ & $\begin{array}{l}\text { Clinical agreement with } \\
\text { expert }\end{array}$ & Moderate & Hau et al. [135] & 150 \\
\hline \multicolumn{9}{|l|}{ Amblyopia } \\
\hline USA & $\begin{array}{l}\text { Multiple clinical trials } \\
{[136,137]}\end{array}$ & 2013 & Ophthalmologist & $\begin{array}{l}\text { Guidelines } \\
\text { adherence }\end{array}$ & Record review & Moderate & Jin et al. [138] & 123 \\
\hline \multicolumn{9}{|l|}{ Esotropia } \\
\hline USA & $\begin{array}{l}\text { Clinical practice } \\
\text { guidelines [139] }\end{array}$ & 2010 & Ophthalmologist & $\begin{array}{l}\text { Guidelines } \\
\text { adherence }\end{array}$ & Record review & Weak & $\begin{array}{l}\text { Gupta et al. } \\
{[140]}\end{array}$ & 200 \\
\hline \multicolumn{9}{|c|}{ Non-infectious uveitis } \\
\hline USA & $\begin{array}{l}\text { Clinical practice } \\
\text { guidelines [141] }\end{array}$ & 2011 & $\begin{array}{l}\text { Ophthalmologist } \\
\text { \& rheumatologist }\end{array}$ & $\begin{array}{l}\text { Current } \\
\text { practice } \\
\text { pattern }\end{array}$ & $\begin{array}{l}\text { Record review and } \\
\text { practitioner survey }\end{array}$ & Moderate & $\begin{array}{l}\text { Nguyen et al. } \\
\text { [142] }\end{array}$ & 580 \\
\hline
\end{tabular}

were met. ${ }^{b}$ Response rate reported in bracket where applicable

with three quality indicators sampled the appropriateness of 'patient education' in diabetes eye care at a single site and reported a below 50\% appropriateness of 'patient education' overall.
Appropriateness of eye care delivery has been measured for cataract, age-related macular degeneration, preventative eye care and five other ocular conditions in 18 separate articles (Table 2). Eye care appropriateness 


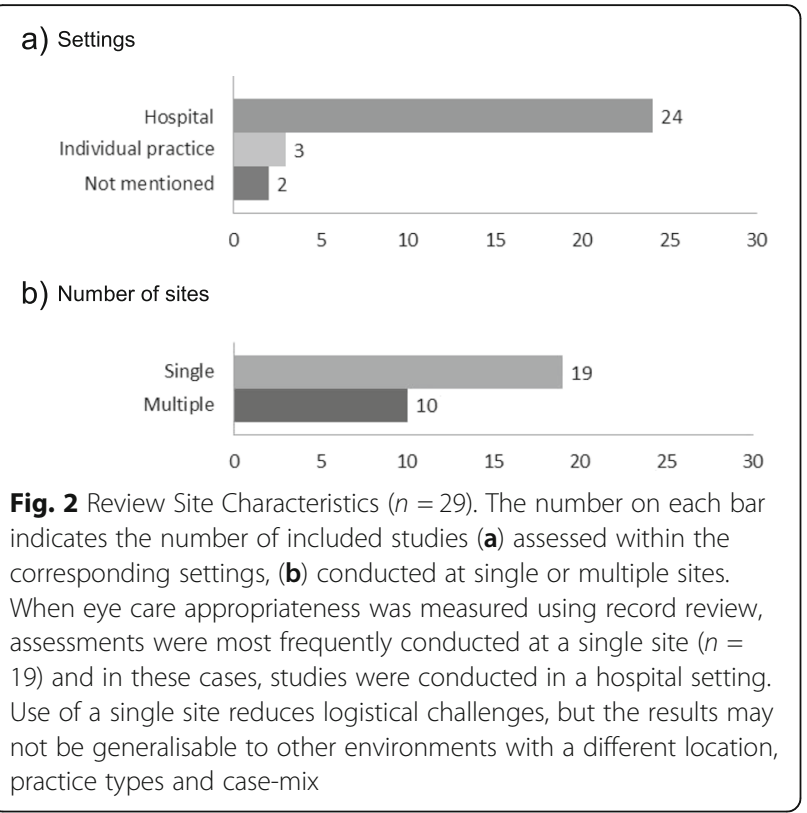

also ranged widely in those studies, for example from 0 to $100 \%$ for dry eye care [134] and for the referral of cataract surgery [107].

Very few studies examined or reported on factors that can modulate appropriateness of eye care delivery. Modifiable factors that have been shown to impact appropriateness of eye care delivery include data entry system (i.e. electronic or paper records) [134], health insurance coverage [76], higher eye care provider density [76], awareness of clinical practice guidelines availability [142], procedural confidence and therapeutic endorsement of optometrists [56] and specialty training conducted in a supportive environment [43]. Nonmodifiable factors that may impact appropriateness of eye care include the severity of patients' eye condition [71], patient's age and ethnicity [54], and practitioner's age [72, 129], gender [129] and years of experience [88]. These factors must therefore be measured and controlled for in any future studies assessing the appropriateness of eye care delivery.

\section{Discussion}

This systematic literature review summarises studies reporting the process of eye care delivery in many different countries using existing published evidence and/or experts' practice to measure appropriateness of eye care. The appropriateness of eye care delivered was found to vary widely for the most commonly reported conditions (glaucoma and diabetic eye care) from 0 to $100 \%$. Appropriate 'management' and 'recall period' for glaucoma were observed. Record review was most commonly used to assess the appropriateness of eye care delivery; this may be explained by the ease of administration and low cost associated with this method, especially when conducted at a single site.

The methodological quality was rated as moderate on average across all methods. Different quality assessment tools were used for to appraise studies with different study design, where some criteria were the same between tools. With consideration of the variety of the study designs and the total numbers of included studies, it was considered beneficial to use a modified quality assessment tool with all questions sourced from existing validated critical appraisal tools (Additional file 2). The quality of the included studies should not be different when different tools are used, when the studies are assessed against the same questions from the existing validated critical appraisal tools.

Comparison of the overall appropriateness of eye care versus the appropriateness for individual domains of eye care between studies presented some challenges for the following reasons:

1) Differences in the number of quality indicators used. Seven quality indicators were used in the Zebardast et al. [48] study, but 19 quality indicators were used by Ong et al. [50] Although both studies assessed appropriateness of eye care against the same glaucoma guidelines, the overall result cannot be easily compared, unless this is done by comparing appropriateness of care of individual quality indicators used by both studies.

2) Differences in eligibility criteria and time frame of quality indicators. Quigley et al. [52] assessed whether practitioners have performed gonioscopy at least once within the previous 6 years for all patients with open-angle glaucoma and found that appropriate care was delivery only $50 \%$ of the time. Conversely, Ong et al. [50] reported 90\% appropriate care for performing gonioscopy on indication. A possible conclusion may be that practitioners in the latter study performed much better than in the former. However, careful observation of the study population characteristics reveals that this appropriateness of care results simply reflects how often practitioners perform gonioscopy in open angle glaucoma in the first instance and use of gonioscopy in cases with a suspicious angle in the latter study.

3) Differences in time interval. Chawla et al. [27] assessed both planned and actual review interval for glaucoma against the guidelines whereas Ong et al. [50] only assessed if the planned follow-up complied with guidelines.

4) Different aspects of the quality indicator are assessed. Appropriateness of 'referral' can be considered in terms of the appropriateness of the referral criteria, the timing of the referral or in 


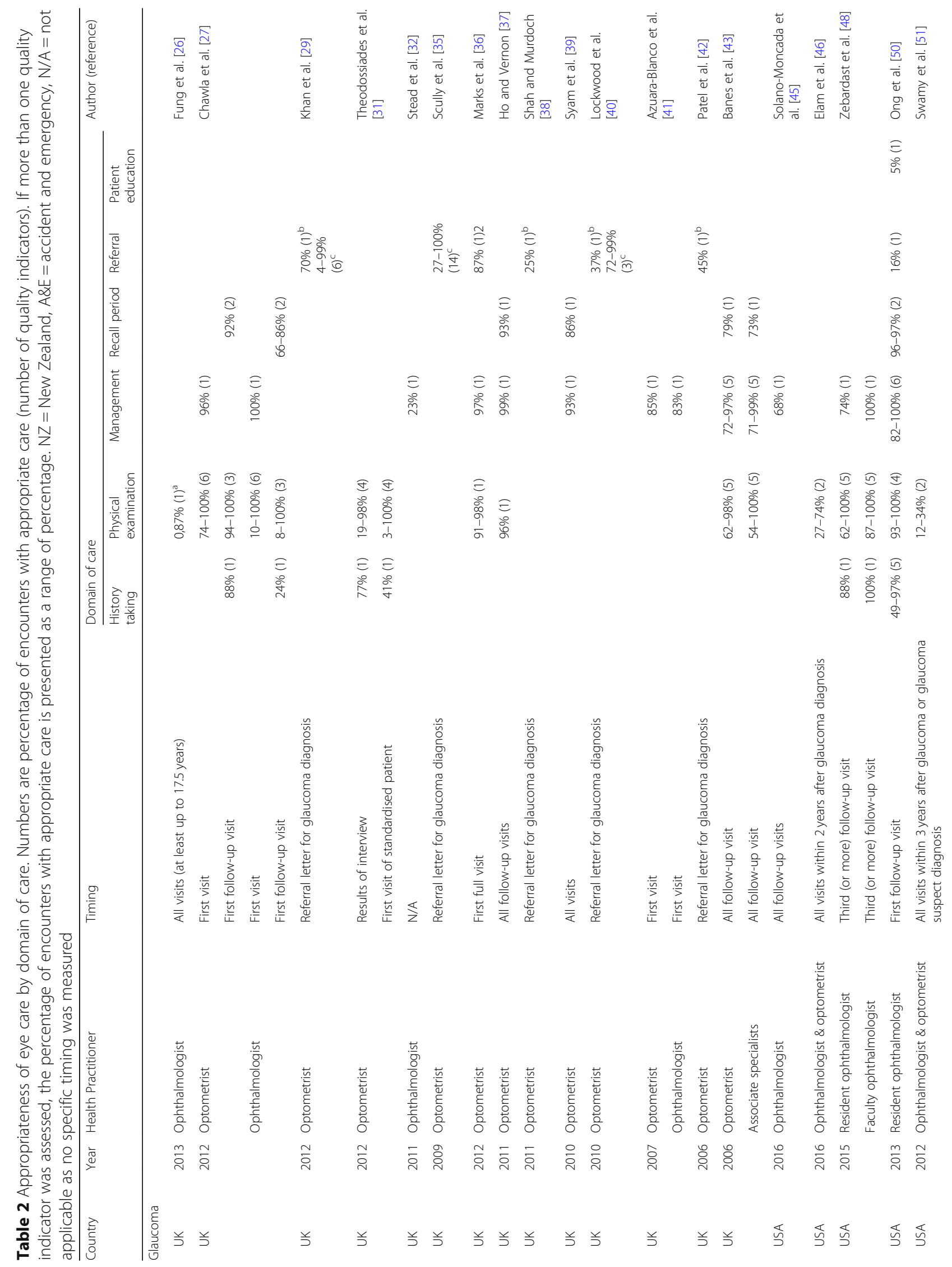




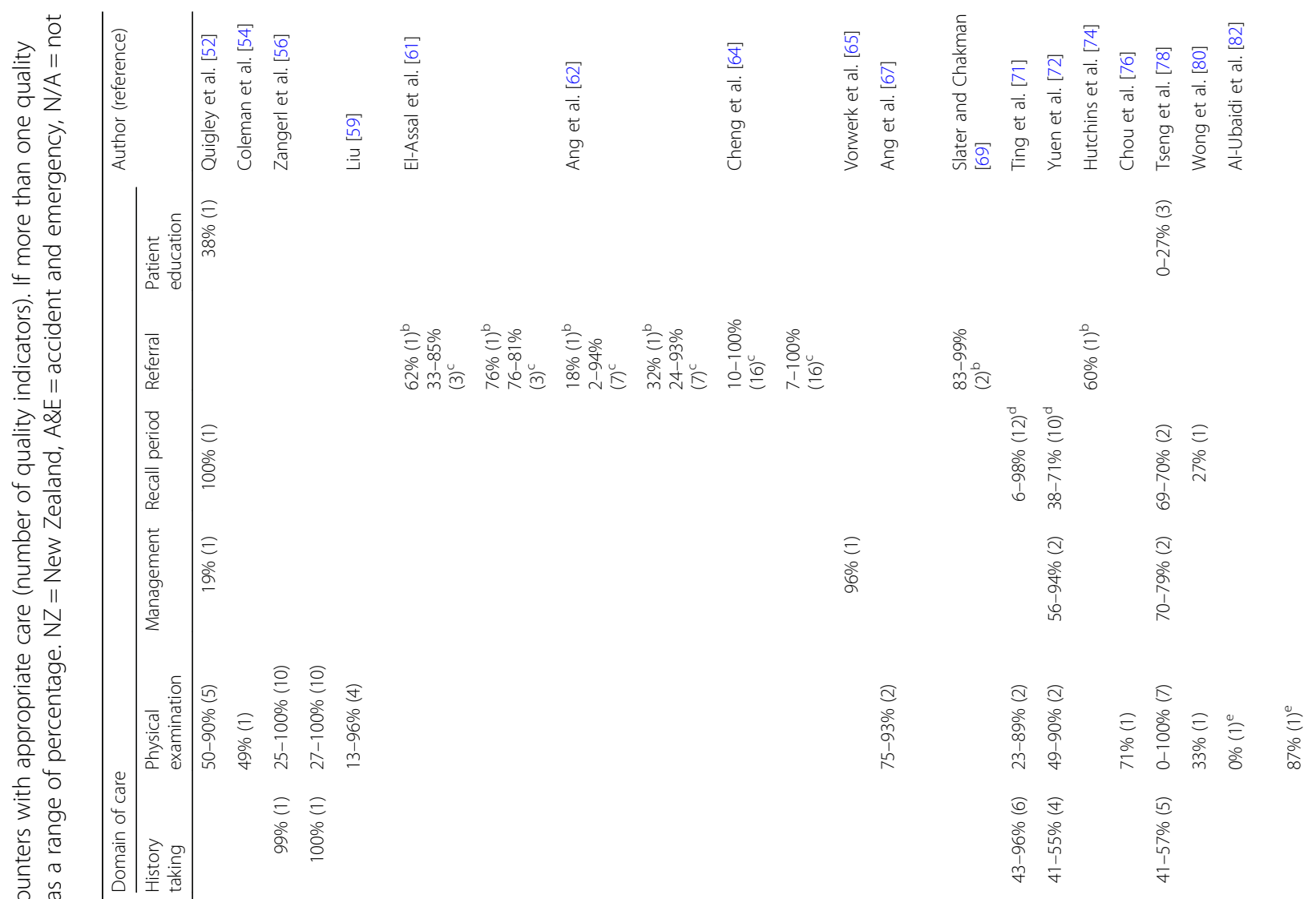

政

के

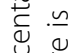

ฮั

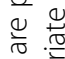

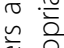

है $\frac{0}{\frac{0}{0}}$

$z$ 요

这家

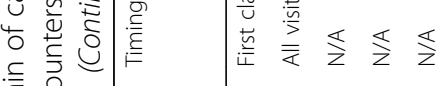

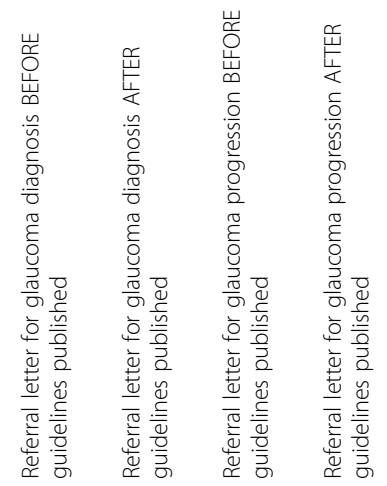

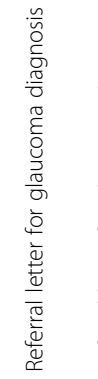

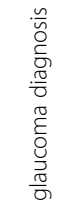

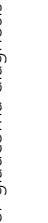

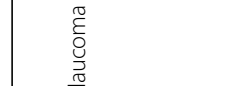

政

운

حิ \&

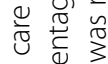

送

बे बें

$\widetilde{u}+$

造

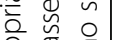

응

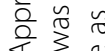

흥

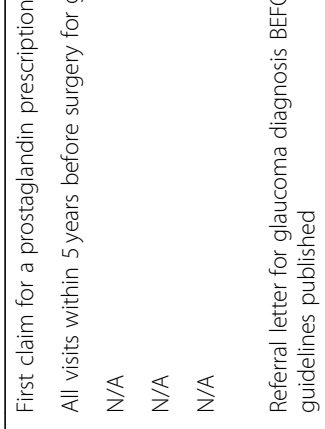

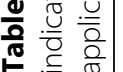

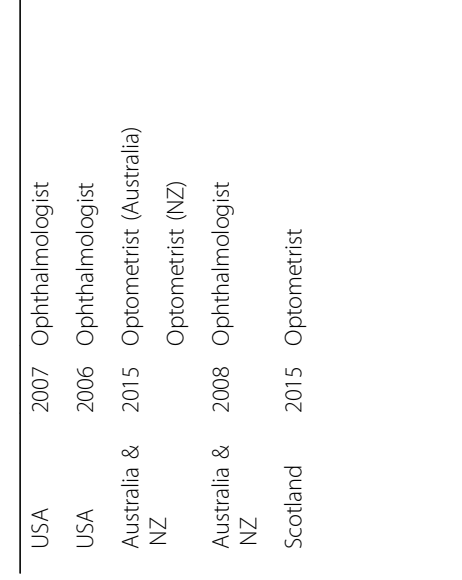

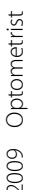

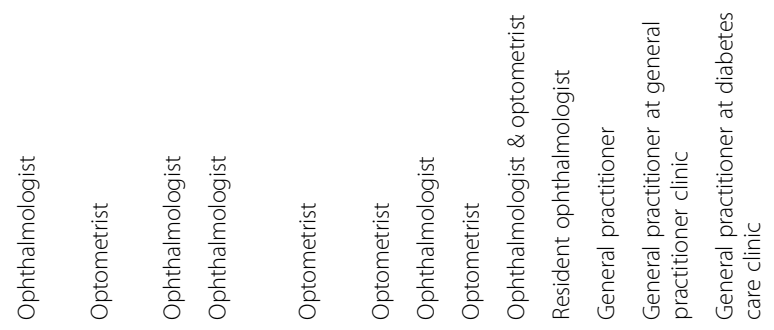

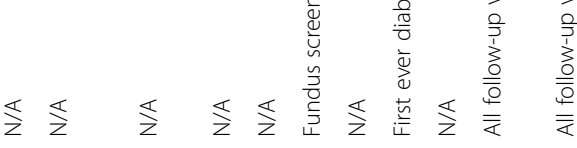

ळे

$\stackrel{4}{\stackrel{4}{2}}$

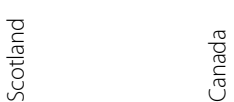

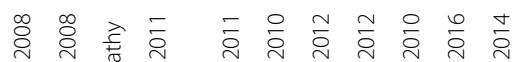

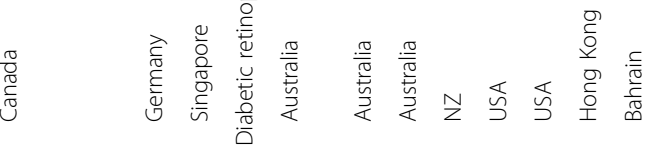




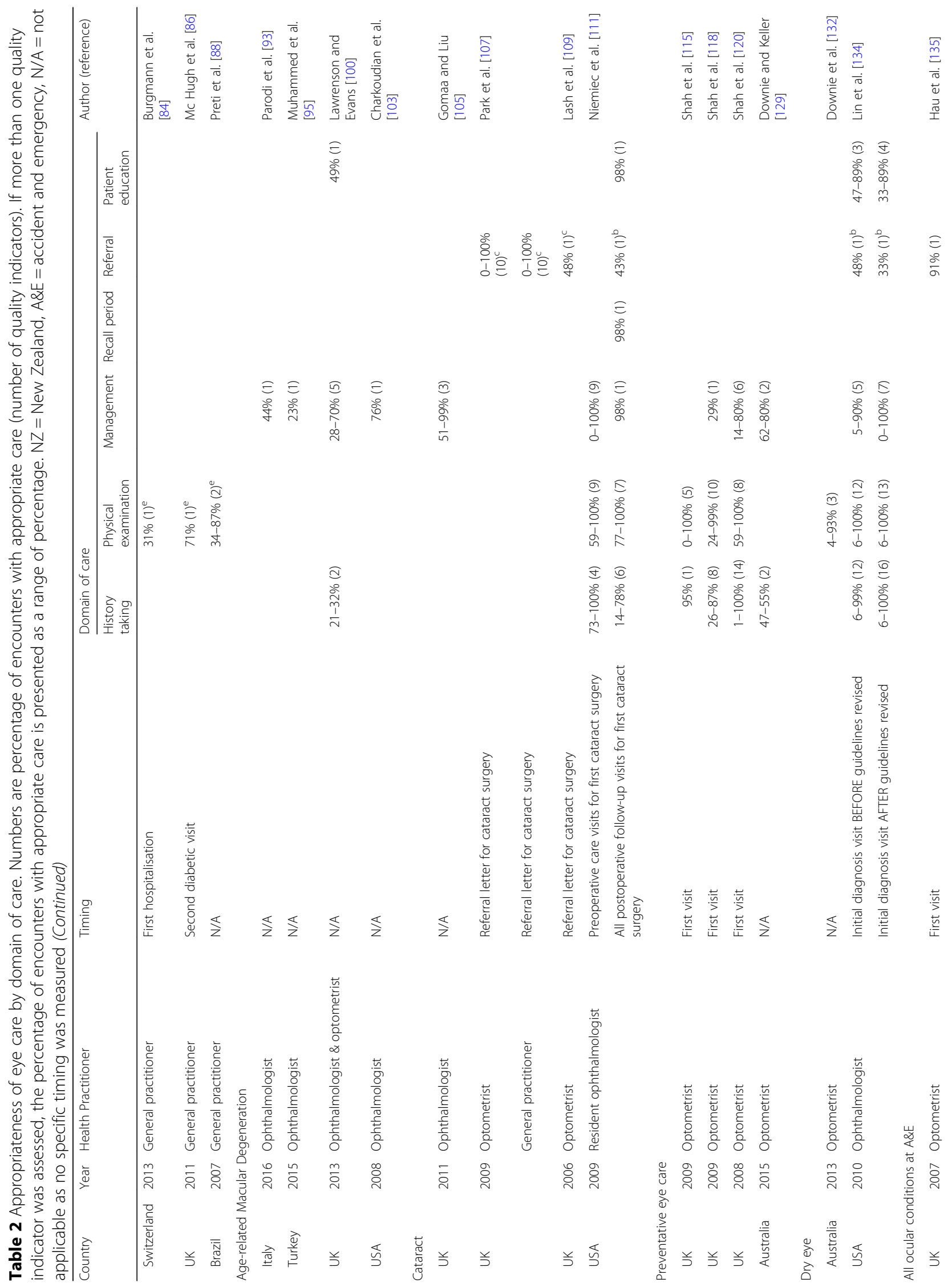




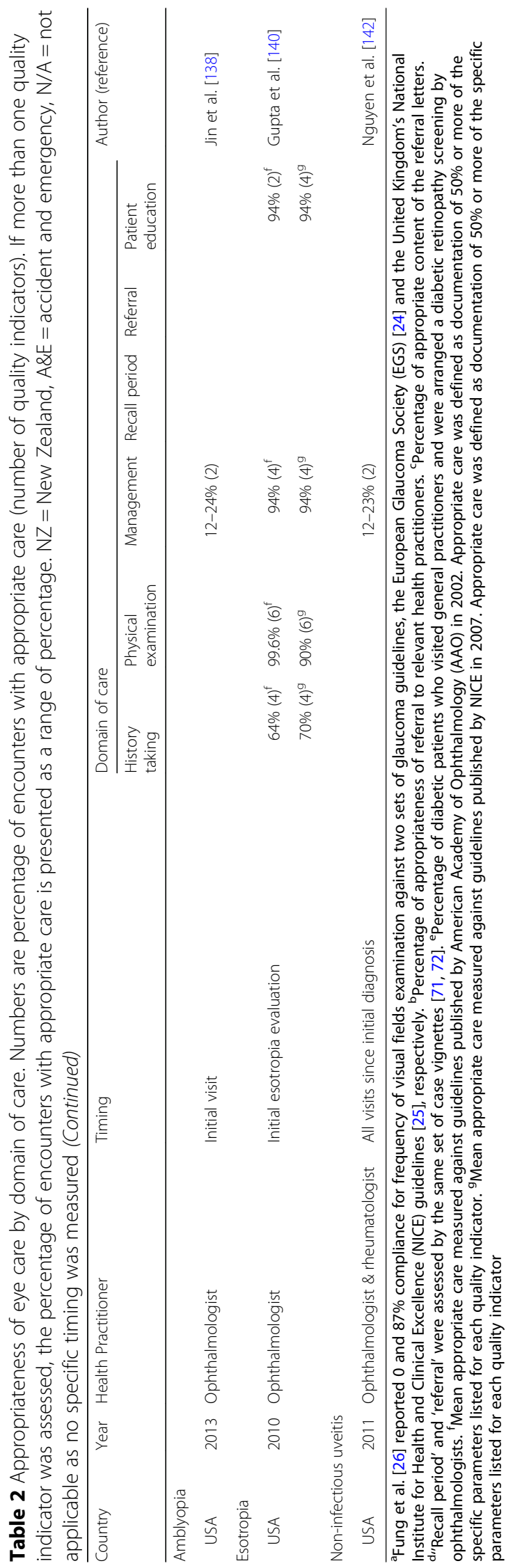




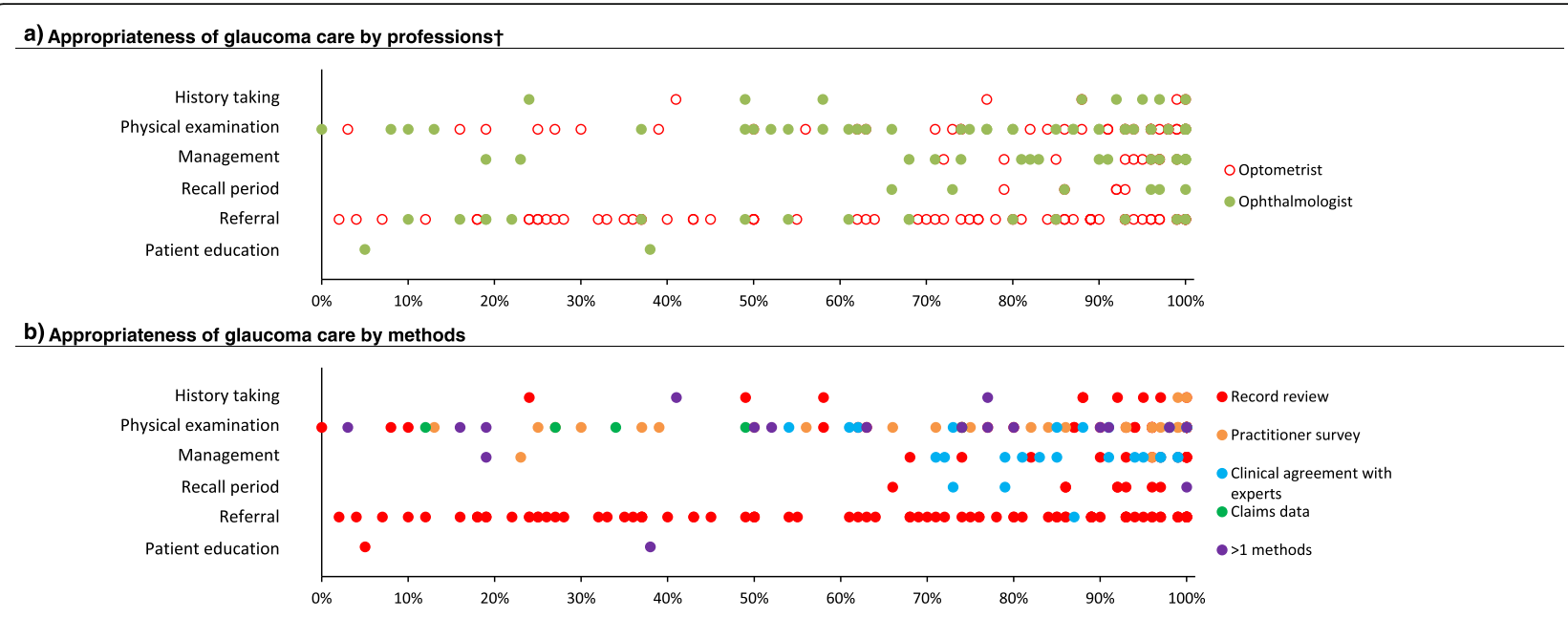

c) Appropriateness of diabetic eye care by professions

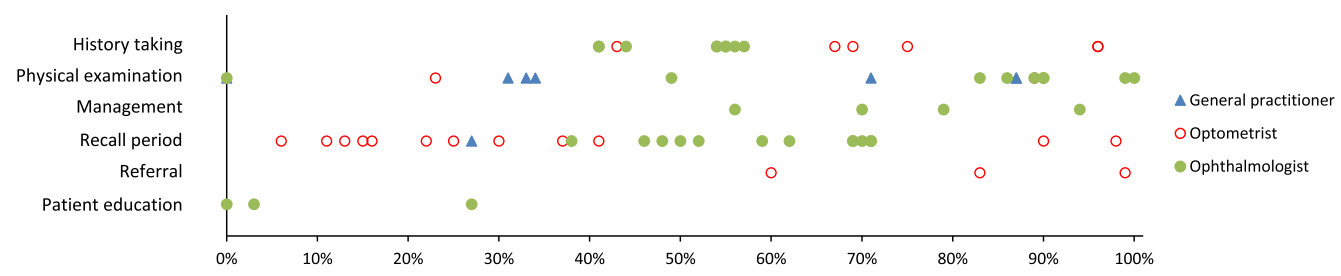

d) Appropriateness of diabetic eye care by methods

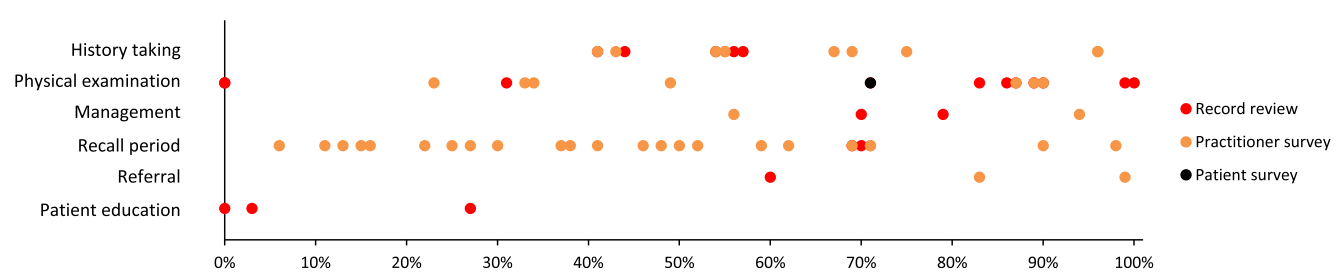

†Results of two studies $[66,69]$ assessed both professions were excluded from this plot.

Fig. 3 Appropriateness of eye care for glaucoma $(\mathbf{a}, \mathbf{b})$ and diabetic retinopathy $(\mathbf{c}, \mathbf{d})$ for various domains of care by profession $(\mathbf{a}, \mathbf{c})$ and methods $(\mathbf{b}, \mathbf{d})$. All quality indicators from the included studies were pooled together. Each data point represented the percentage of compliance against a quality indicator. a Overall, the appropriateness of glaucoma care ranged widely from 2 to $100 \%$. The appropriateness of glaucoma delivered by optometrists and ophthalmologists appeared similar. $\mathbf{b}$ When appropriateness of glaucoma care was assessed using clinical agreement with experts, care was delivered appropriately at least 50\% of the time. The appropriateness of glaucoma care assessed using other methods ranged more widely. $\mathbf{c}$, $\mathbf{d}$ The appropriateness of diabetic eye care ranged widely from 0 to $100 \%$. The wide range and the relatively small number of studies measuring appropriateness of diabetic eye care limited our ability to detect obvious patterns in individual domains for diabetes care

terms of the appropriateness and contents of referral letters. Appropriateness of referral often describes whether patients were referred to the correct people or facilities. Appropriateness and contents of referral letters typically considers if the referral letters contained the required information, according to guidelines or specialist's opinions. However, the percentage of appropriate care of these two aspects may not directly be comparable. Appropriateness of referral pathway or criteria is not necessarily equivalent to an appropriate referral letter and vice versa. For example, Ang et al. [62] reported that the appropriateness of referral letters from optometrists referring for glaucoma progression was $32 \%$ whereas the appropriateness and contents of their referral letters exhibited 24-93\% compliance against the seven quality indicators used.

5) Differences in quality indicator weighing. Most studies weighed all quality indicators evenly, but some assigned different weightings for different quality indicators. Quigley et al. [52] assigned weighting $(0,1,2$ or 3$)$ according to the imputed importance of individual items. Gupta et al. [140] defined appropriate care as the practitioners documenting $50 \%$ or more of the sub-indicators listed for each element. For example, once 2 or 
more of the 4 sub-indicators (frequency of deviation, date of onset, and presence of diplopia or squint) of ocular signs and symptoms were documented, this quality indicator was counted as compliant.

The findings of this systematic review are limited by the lack of a standardised method to measure and report the appropriateness of eye care delivery. The extent to which eye care appropriateness may have been under or overestimated may be significantly influenced by the choice of method used to assess care delivery in these studies. Two-thirds of the included articles measured compliance against recommendations from clinical practice guidelines, which are likely to have been developed using similar evidence sources. In this review, this is likely to have manifested as reporting the appropriateness of eye care according to a somewhat narrow evidence base. However, clinical practice guidelines are primarily developed for and made available to clinicians for the purposes of guiding evidence-based care, which lends credibility to their use as a compliance tool. In addition, studies conducted in one country might not reflect the appropriateness of eye care received in a different country where the health care and education systems, values and expectations could be significantly different [144]. Given that and the diversity of countries where eye care appropriateness has been measured, the generalisability of the various reported findings to other countries is uncertain.

\section{Conclusion}

Studies reporting the appropriateness of eye care delivery in Australia and other developed mainly English-speaking countries, indicated a wide range of appropriateness of care delivery, for glaucoma and diabetic eye care. Existing eyerelated studies have assessed a single condition, a sample from a single centre or a single domain of care even as specific as only one examination technique such as gonioscopy. Consequently, none of the studies identified in the literature review attempted to measure the overall appropriateness of care provided in eye care. One important purpose of measuring appropriateness of care is to help policy makers to allocate limited health resources. Future research would benefit from a more comprehensive approach where appropriateness of eye care delivery is measured across multiple conditions with a single methodology to guide priorities within eye care delivery and monitor quality improvement initiatives.

\section{Additional files}

Additional file 1: Full electronic search strategy for Medline. (DOCX $15 \mathrm{~kb}$ )
Additional file 2: Quality assessment tool. (DOCX $22 \mathrm{~kb}$ )

Additional file 3: Results of quality appraisal of included studies. (DOCX $52 \mathrm{~kb}$ )

\begin{abstract}
Abbreviation
A\&E: Accident and emergency; AAO: American Academy of Ophthalmology; CASP: Critical Appraisal Skills Programme; CINAHL: Cumulative Index to Nursing and Allied Health Literature; EGS: European Glaucoma Society; EPHPP: Effective Public Health Practice Project; JBI: Joanna Briggs Institute; N/ A: Not applicable; NICE: National Institute for Health and Clinical Excellence; $\mathrm{NIH}$ : National Institutes of Health; NZ: New Zealand; PRISMA: Preferred Reporting Items for Systematic reviews and Meta-Analyses; UK: United Kingdom; USA: United States of America
\end{abstract}

\section{Acknowledgements}

We thank UNSW Library, Academic Services Team for their contribution on reviewing the search strategy and conducting the test run.

\section{Authors' contributions}

KCH contributed to search, study design, study selection, data extraction, quality assessment, data analysis, and writing the report. FS contributed to search, study design, quality assessment, data analysis, and critical review. LW contributed to study design and critical review. PH contributed to study design and critical review. SA contributed to quality assessment. AW contributed to study design and critical review. IJ contributed to search, study design, quality assessment, data analysis, and critical review. All authors read and approved the final manuscript.

\section{Funding}

This work was supported by a UNSW Sydney Tuition Fee Scholarship [to $\mathrm{KCH}$; the Saudi Arabian Ministry of Higher Education [to SA]; a UNSW Sydney Faculty of Science June Griffith Fellowship [to IJ]; and a UNSW Sydney Faculty of Science Research Program Grant. The funders of the study had no role in study design, data collection, data analysis, data interpretation, or writing of the report. The corresponding author had full access to all the data in the study and had final responsibility for the decision to submit for publication.

\section{Availability of data and materials}

The datasets generated and/or analysed during the current study are available in the Zenodo repository, DOI: (https://doi.org/10.5281/zenodo. 2597710).

Ethics approval and consent to participate Not applicable.

\section{Consent for publication}

Not applicable.

\section{Competing interests}

The authors declare that they have no competing interests.

\section{Author details}

${ }^{1}$ School of Optometry and Vision Science, UNSW Sydney, Sydney, NSW 2052, Australia. ${ }^{2}$ Eye Health, Injury Division, The George Institute for Global Health, Sydney, Australia. ${ }^{3}$ Faculty of Medicine and Health Sciences, Australian Institute of Health Innovation, Macquarie University, Sydney, New South Wales, Australia. ${ }^{4}$ Centre for Population Health Research, School of Health Sciences, University of South Australia, Adelaide, South Australia, Australia. ${ }^{5}$ Optometry and Vision Science Department, King Saud University, Riyadh, Saudi Arabia. ${ }^{6}$ Save Sight Institute, University of Sydney, Westmead Hospital, Sydney, New South Wales, Australia. ${ }^{7}$ Centre for Vision Research, Westmead Institute for Medical Research, University of Sydney, Westmead Hospital, Sydney, New South Wales, Australia. 


\section{Received: 8 May 2019 Accepted: 29 August 2019} Published online: 06 September 2019

\section{References}

1. Pascolini D, Mariotti SP. Global estimates of visual impairment: 2010. Br J Ophthalmol. 2012;96(5):614-8.

2. Resnikoff $S$, Keys TU. Future trends in global blindness. Indian J Ophthalmol. 2012;60(5):387-95.

3. Voleti VB, Hubschman JP. Age-related eye disease. Maturitas. 2013;75(1):29-33.

4. Konyama K. Essential components of primary eye care. Community Eye Health. 1998;11(26):19-21.

5. Misra V, Vashist P, Malhotra S, Gupta SK. Models for primary eye care services in India. Indian J Community Med. 2015:40(2):79-84.

6. Irving EL, Harris JD, Machan CM, et al. Value of routine eye examinations in asymptomatic patients. Optom Vis Sci. 2016;93(7):660-6.

7. Shah R, Edgar DF, Evans BJ. A comparison of standardised patients, record abstraction and clinical vignettes for the purpose of measuring clinical practice. Ophthalmic Physiol Opt. 2010;30(3):209-24.

8. Slade SV, Davey CJ, Shickle D. Can data in optometric practice be used to provide an evidence base for ophthalmic public health? Ophthalmic Physiol Opt. 2016;36(4):503-11

9. National Health Information Standards and Statistics Committee. The National Health Performance Framework. 2nd ed; 2009.

10. Claiborne Johnston S, Hauser SL. T2 clinical research: judgment day. Ann Neurol. 2008;63(3):A15-6.

11. Berg AO, Atkins D, Tierney W. Clinical practice guidelines in practice and education. J Gen Intern Med. 1997;12(Suppl 2):S25-33.

12. Runciman WB, Hunt TD, Hannaford NA, et al. CareTrack: assessing the appropriateness of health care delivery in Australia. Med J Aust. 2012;197(2): 100-5.

13. McGlynn EA, Asch SM, Adams J, et al. The quality of health care delivered to adults in the United States. N Engl J Med. 2003;348(26):2635-45.

14. Feussner JR, Kizer KW, Demakis JG. The Quality Enhancement Research Initiative (QUERI): from evidence to action. Med Care. 2000; 38(6 Suppl 1):11-6.

15. Demakis JG, McQueen L, Kizer KW, Feussner JR. Quality Enhancement Research Initiative (QUERI): A collaboration between research and clinical practice. Med Care. 2000;38(6 Suppl 1):117-25.

16. Moher D, Liberati A, Tetzlaff J, Altman DG, Grp P. Preferred reporting items for systematic reviews and meta-analyses: The PRISMA statement. Plos Med. 2009;6(7):e1000097.

17. Access Economics. Clear Focus-The Economic Impact of Vision Loss in Australia in 2009: A Report Prepared by Access Economics Pty Limited. Melbourne: Vision 2020 Australia; 2010.

18. Jencks SF, Huff ED, Cuerdon T. Change in the quality of care delivered to Medicare beneficiaries, 1998-1999 to 2000-2001. JAMA. 2003;289(3):305-12

19. Dyba T, Dingsoyr T, Hanssen GK. Applying Systematic Reviews to Diverse Study Types: An Experience Report. In: First International Symposium on Empirical Software Engineering and Measurement (ESEM 2007). Madrid: IEEE Xplore; 2007. p. 225-34.

20. Critical Appraisal Skills Programme. CASP diagnostic checklist. 2013. 03rd Mar 2017 [cited 2013. Available from: http://www.casp-uk.net/checklists.

21. National Institutes of Health. Quality Assessment Tool For Observational Cohort And Cross-Sectional Studies. 2014

22. Joanna Briggs Institute. JBI Critical Appraisal Checklist For Studies Reporting Prevalence Data. 2014.

23. Effective Public Health Practice Project. Quality assessment tool for quantitative studies. 2010. 03rd Mar 2017 [cited 2010. Available from: http:// www.ephpp.ca/tools.html.

24. European Glaucoma Society. Terminology and guidelines for Glaucoma. 3rd ed. Savona: Editrice Dogma; 2008.

25. National Institute for Health and Clinical Excellence. Diagnosis and Management of Chronic Open Angle Glaucoma and Ocular Hypertension. In: NICE Clinical Guideline 85. Manchester: NICE; 2009.

26. Fung SS, Lemer C, Russell RA, Malik R, Crabb DP. Are practical recommendations practiced? A national multi-Centre cross-sectional study on frequency of visual field testing in glaucoma. Br J Ophthalmol. 2013; 97(7):843-7.

27. Chawla A, Patel I, Yuen C, Fenerty C. Patterns of adherence to NICE glaucoma guidance in two different service delivery models. Eye. 2012; 26(11):1412-7.
28. The College of Optometrists and The Royal College of Ophthalmologists. Guidance on the referral of glaucoma suspects by community optometrists: issued by the College of Optometrists and the Royal College of Ophthalmologist. 2009; updated December 2010.2010. 27th Nov 2017 [cited 2010. Available from: https://www.rcophth.ac.uk/wp-content/uploads/2 014/12/2010_PROF_097-Guidance-referral-Glaucoma-suspects-byoptometrists-Dec-2010.pdf.

29. Khan S, Clarke J, Kotecha A. Comparison of optometrist glaucoma referrals against published guidelines. Ophthalmic Physiol Opt. 2012;32(6):472-7.

30. College of optometrists. Code of ethics and guidelines for professional conduct, section D3 examining patients at risk from glaucoma. 2011. 07th Jun 2011 [cited 2011. Available from: http://arch.oucs.ox.ac.uk/ detail/27747/index.html.

31. Theodossiades J, Myint J, Murdoch IE, Edgar DF, Lawrenson JG. Does optometrists' self-reported practice in glaucoma detection predict actual practice as determined by standardised patients? Ophthalmic Physiol Opt. 2012;32(3):234-41

32. Stead R, Azuara-Blanco A, King AJ. Attitudes of consultant ophthalmologists in the UK to initial management of glaucoma patients presenting with severe visual field loss: a national survey. Clin Exp Ophthalmol. 2011;39(9):858-64.

33. General Optical Council. The General Optical Council (injury or disease of the eye and contact lens (qualifications)) (amendment) rules order of council 2005. 2005. 29th Feb 2016 [cited 2005. Available from: http://www. legislation.gov.uk/uksi/2005/1476/made.

34. College of Optometrists. Framework for optometric referrals. 2005. 29th Feb 2016 [cited 2005. Available from: http://www.college-optometrists.org/ membersarea/extranet/OptometricReferrals.pdf.

35. Scully ND, Chu L, Siriwardena D, Wormald R, Kotecha A. The quality of optometrists' referral letters for glaucoma. Ophthalmic Physiol Opt. 2009; 29(1):26-31.

36. Marks JR, Harding AK, Harper RA, et al. Agreement between specially trained and accredited optometrists and glaucoma specialist consultant ophthalmologists in their management of glaucoma patients. Eye. 2012;26(6):853-61.

37. Ho S, Vernon SA. Decision making in chronic glaucoma--optometrists vs ophthalmologists in a shared care service. Ophthalmic Physiol Opt. 2011;31(2):168-73.

38. Shah S, Murdoch IE. NICE - impact on glaucoma case detection. Ophthalmic Physiol Opt. 2011;31(4):339-42.

39. Syam P, Rughani K, Vardy SJ, et al. The Peterborough scheme for community specialist optometrists in glaucoma: a feasibility study. Eye. 2010;24(7):1156-64.

40. >Lockwood AJ, Kirwan JF, Ashleigh Z. Optometrists referrals for glaucoma assessment: a prospective survey of clinical data and outcomes. Eye. 2010; 24(9):1515-9.

41. Azuara-Blanco A, Burr J, Thomas R, Maclennan G, McPherson S. The accuracy of accredited glaucoma optometrists in the diagnosis and treatment recommendation for glaucoma. Br J Ophthalmol. 2007;91(12): 1639-43.

42. Patel UD, Murdoch IE, Theodossiades J. Glaucoma detection in the community: does ongoing training of optometrists have a lasting effect? Eye. 2006;20(5):591-4.

43. Banes MJ, Culham LE, Bunce C, Xing W, Viswanathan A, Garway-Heath D. Agreement between optometrists and ophthalmologists on clinical management decisions for patients with glaucoma. Br J Ophthalmol. 2006;90(5):579-85.

44. American Academy of Ophthalmology Preferred Practice Pattern Glaucoma Panel. Preferred Practice Pattern ${ }^{\oplus}$ Guidelines. In: Primary Open-Angle Glaucoma. San Francisco: American Academy of Ophthalmology; 2010.

45. Solano-Moncada F, Dymerska M, Jefferys JL, Quigley HA. Adherence with the use of target intraocular pressure for glaucoma patients in a large university practice. J Glaucoma. 2016;25(4):e424-32.

46. Elam AR, Blachley TS, Stein JD. Geographic variation in the use of diagnostic testing of patients with newly diagnosed open-angle Glaucoma. Ophthalmology. 2016;123(3):522-31.

47. American Academy of Ophthalmology Preferred Practice Pattern Glaucoma Panel. Preferred Practice Pattern ${ }^{\circledR}$ Guidelines. In: Primary Open-Angle Glaucoma. San Francisco: American Academy of Ophthalmology; 2005.

48. Zebardast N, Solus JF, Quigley HA, Srikumaran D, Ramulu PY. Comparison of resident and glaucoma faculty practice patterns in the care of open-angle glaucoma. BMC Ophthalmol. 2015;15:41. 
49. American Academy of Ophthalmology Preferred Practice Pattern Glaucoma Panel. Preferred Practice Pattern ${ }^{\oplus}$ Guidelines. In: Primary Open-Angle Glaucoma. San Francisco: American Academy of Ophthalmology; 2003.

50. Ong SS, Sanka K, Mettu PS, et al. Resident compliance with the American Academy of Ophthalmology Preferred Practice Pattern guidelines for primary open-angle glaucoma. Ophthalmology. 2013;120(12):2462-9.

51. Swamy L, Smith S, Radcliffe NM. Optic nerve complex imaging in glaucoma Medicare beneficiaries. Ophthalmic Epidemiol. 2012;19(4):249-55.

52. Quigley HA, Friedman DS, Hahn SR. Evaluation of practice patterns for the care of open-angle glaucoma compared with claims data: the Glaucoma adherence and persistency study. Ophthalmology. 2007;114(9):1599-606.

53. American Academy of Ophthalmology Preferred Practice Patterns Committee Glaucoma Panel. Preferred Practice Pattern ${ }^{\oplus}$ Guidelines. In: Primary Angle Closure Glaucoma. San Francisco: American Academy of Ophthalmology; 1996.

54. Coleman AL, Yu F, Evans SJ. Use of gonioscopy in Medicare beneficiaries before glaucoma surgery. J Glaucoma. 2006;15(6):486-93.

55. National Health and Medical Research Council. NHMRC guidelines for the screening, prognosis, diagnosis, management and prevention of glaucoma. 2010. 29th Jul 2015 [cited 2010. Available from: https://www.nhmrc.gov.au/_ files_nhmrc/publications/attachments/cp113_glaucoma_120404.pdf.

56. Zangerl B, Hayen A, Mitchell P, Jamous KF, Stapleton F, Kalloniatis M. Therapeutic endorsement enhances compliance with national glaucoma guidelines in Australian and New Zealand optometrists. Ophthalmic Physiol Opt. 2015;35(2):212-24.

57. European Glaucoma Society. Terminology and guidelines for Glaucoma. 2nd ed. Savona: Editrice Dogma; 2003.

58. South East Asia Glaucoma Interest Group. Asia Pacific Glaucoma Guidelines. Singapore: South East Asia Glaucoma Interest Group; 2003.

59. Liu L. Australia and New Zealand survey of glaucoma practice patterns. Clin Exp Ophthalmol. 2008;36(1):19-25.

60. National Health Service National Service Scotland. General Ophthalmic Services Eye Care. 2006. 29th Feb 2016 [cited 2006. Available from: http:// www.isdscotland.org/Health-Topics/Eye-Care/General-Ophthalmic-Services.

61. El-Assal K, Foulds J, Dobson S, Sanders R. A comparative study of glaucoma referrals in Southeast Scotland: effect of the new general ophthalmic service contract, Eyecare integration pilot programme and NICE guidelines. BMC ophthalmol. 2015;15:172

62. Ang GS, Ng WS, Azuara-Blanco A. The influence of the new general ophthalmic services (GOS) contract in optometrist referrals for glaucoma in Scotland. Eye (Lond). 2009;23(2):351-5.

63. Canadian Ophthalmological Society Glaucoma Clinical Practice Guideline Expert C, Canadian Ophthalmological S. Canadian Ophthalmological Society evidence-based clinical practice guidelines for the management of glaucoma in the adult eye. Can J Ophthalmol. 2009;44(Suppl 1):S7-93.

64. Cheng J, Beltran-Agullo L, Trope GE, Buys YM. Assessment of the quality of glaucoma referral letters based on a survey of glaucoma specialists and a glaucoma guideline. Ophthalmology. 2014;121(1):126-33.

65. Vorwerk C, Thelen U, Buchholz P, Kimmich F. Treatment of glaucoma patients with insufficient intraocular pressure control: a survey of German ophthalmologists in private practice. Curr Med Res Opin. 2008;24(5):1295-301.

66. Weinreb RN, Friedman DS. Angle closure and angle closure Glaucoma: reports and consensus statements of the 3rd global AIGS consensus meeting on angle closure Glaucoma. Amsterdam: Kugler Publications; 2006.

67. Ang MH, Baskaran M, Kumar RS, et al. National survey of ophthalmologists in Singapore for the assessment and management of asymptomatic angle closure. J Glaucoma. 2008;17(1):1-4

68. National Health and Medical Research Council. Guidelines for the Management of Diabetic Retinopathy. Canberra: National Health \& Medical Research Council; 2008. Available from: http://www.nhmrc.gov.au/_files_ nhmrc/publications/attachments/di15.pdf

69. Slater J, Chakman J. Referral of diabetic macular oedema by Australian optometrists: comment. Clin Exp Ophthalmol. 2011;39(9):923-4.

70. National Health and Medical Research Council. Clinical practice guideline: management diabetic retinopathy. 1997. 29th Feb 2016 [cited 1997. Available from: http://www.nhmrc.gov.au/publications/synopses/cp53covr.htm.

71. Ting DS, Ng JQ, Morlet N, et al. Diabetic retinopathy management by Australian optometrists. Clin Exp Ophthalmol. 2011;39(3):230-5.

72. Yuen J, Clark A, Ng JQ, et al. Further survey of Australian ophthalmologist's diabetic retinopathy management: did practice adhere to National Health and Medical Research Council guidelines? Clin Exp Ophthalmol. 2010;38(6):613-9.
73. Aldington SK, Kohner EM, Meuer S, Klein R, Sjølie AK. Methodology for retinal photography and assessment of diabetic retinopathy: the EURODIAB IDDM complications study. Diabetologia. 1995;38:971-5.

74. Hutchins E, Coppell KJ, Morris A, Sanderson G. Diabetic retinopathy screening in New Zealand requires improvement: results from a multiCentre audit. Aust N Z J Public Health. 2012;36(3):257-62.

75. American Diabetes Association. Standards of medical care in diabetes 2008. Diabetes Care. 2008;31(Suppl 1):S12-54.

76. Chou CF, Zhang X, Crews JE, Barker LE, Lee PP, Saaddine JB. Impact of geographic density of eye care professionals on eye care among adults with diabetes. Ophthalmic Epidemiol. 2012;19(6):340-9.

77. American Academy of Ophthalmology Retinal/Vitreous Panel. Preferred Practice Pattern ${ }^{\oplus}$ Guidelines. In: Diabetic Retinopathy. San Francisco: American Academy of Ophthalmology; 2003. 29th Feb 2016.

78. Tseng VL, Greenberg PB, Scott IU, Anderson KL. Compliance with the American Academy of ophthalmology preferred practice pattern for diabetic retinopathy in a resident ophthalmology clinic. Retina. 2010;30(5):787-94.

79. Wong MC, Sin CK, Lee JP. The reference framework for diabetes care in primary care settings. Hong Kong Med J. 2012;18(3):238-46.

80. Wong MCS, Wang HHX, Kwan MWM, et al. The adoption of the Reference Framework for diabetes care among primary care physicians in primary care settings: A cross-sectional study. Medicine. 2016;95(31):e4108.

81. Ministry of Health. Kingdom of Bahrain. In: Clinical Practice Guidelines on Diabetes Mellitus; 2008. Available from: https:/www.moh.gov.sg/content/moh_ web/healthprofessionalsportal/doctors/guidelines/cpg_medical.html\#withdrawn.

82. Al-Ubaidi BA, Al-Khadraji MA, Al-Hermi B. Measuring adherence rate to quality indicators for diabetes care identified by primary health care in Bahrain. Saudi Med J. 2014;35(9):975-80.

83. Philippe J, Brändle M, Carrel J, Diem P, Keller U, Kuntschen F, Ruiz J, Stahl M, Weissenberger B, Spinas GA. Massnahmen zur blutzuckerkontrolle bei patienten mit typ-2-diabetes-mellitus: consensus statement der schweizerischen gesellschaft für endokrinologie. In: Swiss Medical Forum, vol. 9; 2009. p. 50-5.

84. Burgmann K, Fatio S, Jordi B, Rutishauser J. Medical care of type 2 diabetes mellitus in light of international and national recommendations: a retrospective analysis. Swiss Med Wkly. 2013;143:w13871.

85. Harkins V. A practical guide to integrated type 2 diabetes care. Dublin: National Health Service Executive; 2008.

86. Mc Hugh S, Marsden P, Brennan C, et al. Counting on commitment; the quality of primary care-led diabetes management in a system with minimal incentives. BMC Health Serv Res. 2011;11:348.

87. American Academy of Ophthalmology. Preferred Practice Pattern ${ }^{\oplus}$ Guidelines. In: Diabetic Retinopathy. San Francisco: American Academy of Ophthalmology; 1993.

88. Preti RC, Saraiva F, Junior JA, Takahashi WY, da Silva ME. How much information do medical practitioners and endocrinologists have about diabetic retinopathy? Clinics (Sao Paulo). 2007;62(3):273-8.

89. Age-Related Eye Disease Study Research Group. A randomized, placebocontrolled, clinical trial of high-dose supplementation with vitamins $C$ and $\mathrm{E}$, beta carotene, and zinc for age-related macular degeneration and vision loss: AREDS report no. 8. Arch Ophthalmol. 2001;119(10):1417-36.

90. Age-Related Eye Disease Study 2 Research Group. Lutein + zeaxanthin and omega-3 fatty acids for age-related macular degeneration: the Age-Related Eye Disease Study 2 (AREDS2) randomized clinical trial. JAMA. 2013;309(19):2005-15.

91. Age-Related Eye Disease Study 2 Research Group. Secondary analyses of the effects of lutein/zeaxanthin on age-related macular degeneration progression: AREDS2 report No. 3. JAMA Ophthalmol. 2014;132(2):142-9.

92. Musch DC. Evidence for including lutein and zeaxanthin in oral supplements for age-related macular degeneration. JAMA Ophthalmol. 2014;132(2):139-41.

93. Parodi MB, Zucchiatti I, Cicinelli MV, Cascavilla ML, Bandello F. Nutritional supplementation in age-related macular degeneration. Retina. 2016;36(6):1119-25.

94. Fletcher AE, Bentham GC, Agnew M, et al. Sunlight exposure, antioxidants, and age-related macular degeneration. Arch Ophthalmol. 2008;126(10):1396-403.

95. Muhammed S, Yuksel H, Sahin A, et al. Approach of Turkish ophthalmologists to micronutrition in age-related macular degeneration. Arq Bras Oftalmol. 2015;78(1):10-4.

96. Chakravarthy U, Wong TY, Fletcher A, et al. Clinical risk factors for agerelated macular degeneration: a systematic review and meta-analysis. BMC Ophthalmol. 2010;10:31.

97. Thornton J, Edwards R, Mitchell P, Harrison RA, Buchan I, Kelly SP. Smoking and age-related macular degeneration: a review of association. Eye (Lond). 2005;19(9):935-44. 
98. Mares JA, Moeller SM. Diet and age-related macular degeneration: expanding our view. Am J Clin Nutr. 2006;83(4):733-4.

99. Evans JR, Lawrenson JG. Antioxidant vitamin and mineral supplements for slowing the progression of age-related macular degeneration. Cochrane Database Syst Rev. 2012;11:CD000254.

100. Lawrenson JG, Evans JR. Advice about diet and smoking for people with or at risk of age-related macular degeneration: a cross-sectional survey of eye care professionals in the UK. BMC Public Health. 2013;13:564.

101. Omenn GS, Goodman GE, Thornquist MD, et al. Effects of a combination of beta carotene and vitamin A on lung cancer and cardiovascular disease. N Engl J Med. 1996;334(18):1150-5.

102. Alpha-Tocopherol Beta Carotene Cancer Prevention Study Group. The effect of vitamin $\mathrm{E}$ and beta carotene on the incidence of lung cancer and other cancers in male smokers. N Engl J Med. 1994;330(15):1029-35.

103. Charkoudian LD, Gower EW, Solomon SD, Schachat AP, Bressler NM, Bressler SB. Vitamin usage patterns in the prevention of advanced age-related macular degeneration. Ophthalmology. 2008;115(6):1032-8 e4.

104. The Royal College of Ophthalmologists. Cataract Surgery Guidelines. Updated 2007. London: Royal College of Ophthalmologists; 2004.

105. Gomaa A, Liu C. Nd:YAG laser capsulotomy: a survey of UK practice and recommendations. Eur J Ophthalmol. 2011;21(4):385-90.

106. College of Optometrists. Framework for the optometric co-management of patients with cataracts. 2003

107. Park JC, Ross AH, Tole DM, Sparrow JM, Penny J, Mundasad MV. Evaluation of a new cataract surgery referral pathway. Eye. 2009;23(2):309-13.

108. National Health Service Executive. Action on cataracts. Good practice guidance. London: Department of Health; 2000. Available from: https://www.rcophth.ac.uk/ wp-content/uploads/2015/03/Action-on-cataracts-Jan-2000-dh_4014514.pdf

109. Lash SC, Prendiville CP, Samson A, Lewis K, Munneke R, Parkin BT. Optometrist referrals for cataract and "action on cataracts" guidelines: are optometrists following them and are they effective? Ophthalmic Physiol Opt. 2006;26(5):464-7.

110. American Academy of Ophthalmology Cataract and Anterior Segment Panel. Preferred Practice Pattern ${ }^{\oplus}$ Guidelines. In: Cataract In The Adult Eye. San Francisco: American Academy of Ophthalmology; 2006

111. Niemiec ES, Anderson KL, Scott IU, Greenberg PB. Evidence-based management of resident-performed cataract surgery: an investigation of compliance with a preferred practice pattern. Ophthalmology. 2009;116(4):678-84.

112. College of Optometrists. What happens in an eye examination? Available from: www.collegeoptometrists.org/index.aspx/pcms/site.Public_Related_ Links.The_eye_examination.What_Happens_in_/.

113. Association of Optometrists. The eye examination. 2008. 25th Apr 2008 [cited 2008. Available from: www.assoc-optometrists.org.

114. College of Optometrists. Code of ethics and guidelines for professional conduct. 2008. 27th May 2008 [cited 2008. Available from: http://www.collegeoptometrists.org/index.aspx/pcms/site.publication.Ethics_Guidelines.recent.

115. Shah R, Edgar DF, Spry PG, et al. Glaucoma detection: the content of optometric eye examinations for a presbyopic patient of African racial descent. Br J Ophthalmol. 2009;93(4):492-6.

116. National Health Service. The General Ophthalmic Services contracts regulations 2008. 2008. 05th May 2016 [cited 2008. Available from: http://www.pcc.nhs.uk/ uploads/Optometry/May\%2008/gos_contracts_regs_2008.pdf.

117. Harvey BF. Routine eye examination. London: Elsevier; 2005.

118. Shah R, Edgar DF, Harle DE, et al. The content of optometric eye examinations for a presbyopic patient presenting with symptoms of flashing lights. Ophthalmic Physiol Opt. 2009;29(2):105-26.

119. General Optical Council. Annual report; 2008. p. 1-44. 01 st Jul 2016 Cited 2008. Available from: http://www.optical.org/goc/filemanager/root/site_ assets/publications/annual_reports/GOC_RepAcc07_2.pdf

120. Shah R, Edgar DF, Rabbetts $R$, et al. The content of optometric eye examinations for a young myope with headaches. Ophthalmic Physiol Opt. 2008;28(5):404-21.

121. Christen WG, Manson JE, Seddon JM, et al. A prospective study of cigarette smoking and risk of cataract in men. JAMA. 1992;268(8):989-93.

122. Hankinson SE, Willett WC, Colditz GA, et al. A prospective study of cigarette smoking and risk of cataract surgery in women. JAMA. 1992;268(8):994-8.

123. Klein $\mathrm{R}$, Klein BE, Linton $\mathrm{KL}$, DeMets $\mathrm{DL}$. The beaver dam eye study: the relation of age-related maculopathy to smoking. Am J Epidemiol. 1993;137(2):190-200.

124. Seddon JM, Willett WC, Speizer FE, Hankinson SE. A prospective study of cigarette smoking and age-related macular degeneration in women. JAMA. 1996;276(14):1141-6.

125. Age-Related Eye Disease Study Research Group. The relationship of dietary carotenoid and vitamin A, E, and C intake with age-related macular degeneration in a case-control study: AREDS Report No. 22. Arch Ophthalmol. 2007;125(9):1225-32.

126. Seddon JM, Ajani UA, Sperduto RD, et al. Dietary carotenoids, vitamins A, C, and $\mathrm{E}$, and advanced age-related macular degeneration. Eye Disease CaseControl Study Group. JAMA. 1994;272(18):1413-20.

127. Augood C, Chakravarthy U, Young I, et al. Oily fish consumption, dietary docosahexaenoic acid and eicosapentaenoic acid intakes, and associations with neovascular age-related macular degeneration. Am J Clin Nutr. 2008:88(2):398-406

128. Merle BM, Delyfer MN, Korobelnik JF, et al. High concentrations of plasma $\mathrm{n} 3$ fatty acids are associated with decreased risk for late age-related macular degeneration. J Nutr. 2013;143(4):505-11.

129. Downie LE, Keller PR. The Self-Reported Clinical Practice Behaviors of Australian Optometrists as Related to Smoking, Diet and Nutritional Supplementation. Plos One. 2015;10(4):e0124533.

130. Management and Therapy Subcommittee of the International Dry Eye WorkShop. Management and therapy of dry eye disease: report of the management and therapy Subcommittee of the International dry eye WorkShop (2007). Ocul Surf. 2007;5(2):163-78.

131. Diagnostic Methodology Subcommittee of the International Dry Eye Workshop. Methodologies to diagnose and monitor dry eye disease: report of the diagnostic methodology Subcommittee of the International dry eye WorkShop (2007). Ocul Surf. 2007;5(2):108-52.

132. Downie LE, Keller PR, Vingrys AJ. An evidence-based analysis of Australian Optometrists' dry eye practices. Optom Vis Sci. 2013;90(12):1385-95.

133. American Academy of Ophthalmology. Preferred Practice Pattern ${ }^{\oplus}$ Guidelines. In: Dry Eye Syndrome. San Francisco: American Academy of Ophthalmology; 1998

134. Lin IC, Gupta PK, Boehlke CS, Lee PP. Documentation of conformance to preferred practice patterns in caring for patients with dry eye. Arch Ophthalmol. 2010;128(5):619-23.

135. Hau S, Ehrlich D, Binstead K, Verma S. An evaluation of optometrists' ability to correctly identify and manage patients with ocular disease in the accident and emergency department of an eye hospital. Br J Ophthalmol. 2007;91(4):437-40

136. Repka MX, Beck RW, Holmes JM, et al. A randomized trial of patching regimens for treatment of moderate amblyopia in children. Arch Ophthalmol. 2003;121(5):603-11.

137. Holmes JM, Kraker RT, Beck RW, et al. A randomized trial of prescribed patching regimens for treatment of severe amblyopia in children. Ophthalmology. 2003;110(11):2075-87.

138. Jin YP, Chow AHY, Colpa L, Wong AMF. Clinical translation of recommendations from randomized clinical trials on patching regimen for amblyopia. Ophthalmology. 2013;120(4):657-62.

139. American Academy of Ophthalmology. Preferred Practice Pattern ${ }^{\oplus}$ Guidelines. In: Esotropia. San Francisco: American Academy of Ophthalmology; 2002.

140. Gupta PK, Freedman SF, Lee PP. Conformance with preferred practice patterns in caring for children with esotropia. J Pediatr Ophthalmol Strabismus. 2010;47(3):145-9 quiz 50-1.

141. Jabs DA, Nussenblatt RB, Rosenbaum JT. Standardization of Uveitis Nomenclature (SUN) Working Group. Standardization of uveitis nomenclature for reporting clinical data. Results of the First International Workshop. Am J Ophthalmol. 2005;140(3):509-16.

142. Nguyen QD, Hatef $E$, Kayen B, et al. A cross-sectional study of the current treatment patterns in noninfectious uveitis among specialists in the United States. Ophthalmology. 2011;118(1):184-90.

143. Andonegui J, Zurutuza A, de Arcelus MP, et al. Diabetic retinopathy screening with non-mydriatic retinography by general practitioners: 2-year results. Prim Care Diabetes. 2012;6(3):201-5.

144. Schoen C, Osborn R, Doty MM, Bishop M, Peugh J, Murukutla N. Toward higher-performance health systems: adults' health care experiences in seven countries, 2007. Health Aff (Millwood). 2007;26(6):w717-34

\section{Publisher's Note}

Springer Nature remains neutral with regard to jurisdictional claims in published maps and institutional affiliations. 